\title{
Article \\ Phragmén-Lindelöf Alternative Results for a Class of Thermoelastic Plate
}

\author{
Shiguang Luo ${ }^{1}$, Jincheng Shi ${ }^{2, *}$ and Baiping Ouyang ${ }^{2}$ \\ 1 Department of Applied Mathematics, Guangdong University of Finance, Yingfu Road, \\ Guangzhou 510521, China; 26-047@gduf.edu.cn \\ 2 School of Data Scinence, Guangzhou Huashang College, Huashang Road, Guangzhou 511300, China; \\ oytengfei79@gdhsc.edu.cn \\ * Correspondence: shijc0818@gdhsc.edu.cn
}

Citation: Luo, S.; Shi, J.; Ouyang, B. Phragmén-Lindelöf Alternative Results for a Class of Thermoelastic Plate. Symmetry 2021, 13, 2256. https:// doi.org/10.3390/sym13122256

Academic Editors: Sergei D. Odintsov and Nicusor Minculete

Received: 1 November 2021

Accepted: 23 November 2021

Published: 26 November 2021

Publisher's Note: MDPI stays neutral with regard to jurisdictional claims in published maps and institutional affiliations.

Copyright: (c) 2021 by the authors. Licensee MDPI, Basel, Switzerland. This article is an open access article distributed under the terms and conditions of the Creative Commons Attribution (CC BY) license (https:// creativecommons.org/licenses/by/ $4.0 /)$.

\begin{abstract}
The spatial properties of solutions for a class of thermoelastic plate with biharmonic operator were studied. The energy method was used. We constructed an energy expression. A differential inequality which the energy expression was controlled by a second-order differential inequality is deduced. The Phragmén-Lindelö $f$ alternative results of the solutions were obtained by solving the inequality. These results show that the Saint-Venant principle is also valid for the hyperbolic-hyperbolic coupling equations. Our results can been seen as a version of symmetry in inequality for studying the Phragmén-Lindelöf alternative results.
\end{abstract}

Keywords: thermoelastic plate; Phragmén-Lindelöf alternative; Saint-Venant principle; biharmonic equation

\section{Introduction}

Saint Venant principle points out that for any equilibrium force system on an elastic body, if its action point is limited to a given ball, the displacement and stress generated by the equilibrium force system at any point where the distance from the load is far greater than the radius of the ball can be ignored. This principle is widely used in engineering mechanics in practice. Many papers in the literature dealt with the study of the Saint-Venant principle. For example, Horgan and Knowles [1] and Horgan [2,3] studied the Saint-Venant principle in different equations and different situations. The traditional characteristic of the Saint-Venant theorem is to derive the energy decay estimates of the solutions. Usually, these decays are exponential with the spatial distance from the finite end to the infinity. In order to have some understandings about the study of the Saint-Venant Principle, one could refer to the papers [4-9]. In recent years, the studies of Saint-Venant principle for hyperbolic or quasihyperbolic equations are abundant. Especially for the studies of the spatial behavior of viscoelasticity equations, we could see papers [10-13]. When the spatial variable tends to infinity, the solution is decreasing. In the research of solution spacial decay estimates, people often need to add the solutions must satisfy some constraints at infinity. Many scholars have begun to study the Phragmén-Lindelöf alternative results of solutions. The advantage of this situation is that there is no need to add constraints on the solutions at infinity. The classical Phragmén-Lindelöf theorem states that the solutions of the harmonic equation must grow exponentially or decay exponentially with distance from the finite end of the cylinder to infinity. Payne and Schaefer [14] extended the study from harmonic equation to biharmonic equation. They obtained the Phragmén-Lindelöf alternative results for biharmonic equation in three different regions. Literatures [15-18] studied the spatial behaviors of biharmonic equations by various methods. In particular, we can see that Liu and Lin [19] studied the spatial properties for time-dependent stokes equation. They transformed the equation to a biharmonic equation and obtained the Phragmén-Lindelöf results by using a second-order differential inequality. The abovementioned studies from the 
literature all consider a single equation. Recently, there some new results about the studies of the hyperbolic equations or biharmonic Equations have been published (see [20-25]). For studies of other equations using energy method, see [26-29].

The domain we consider in this paper is defined as follows:

$$
\Omega_{0}=\left\{\left(x_{1}, x_{2}\right) \mid x_{1}>0,0<x_{2}<h\right\},
$$

with $h$ is a given positive number. We now give the following notation:

$$
L_{z}=\left\{\left(x_{1}, x_{2}\right) \mid x_{1}=z \geq 0,0 \leq x_{2} \leq h\right\} .
$$

In reference [30], the authors studied the coupled system of wave-plate type with thermal effect. They obtained the results of the analytic property and the exponential stability of the $C_{0}$-semigroup. The equations are as follows:

$$
\left\{\begin{array}{l}
\rho_{1} u, t t-\Delta u-\mu \Delta u, t+\lambda \Delta v s .=0 \\
\rho_{2} v_{, t}+\gamma \Delta^{2} v s .+\lambda \Delta u+m \Delta \theta=0 \\
\tau \theta, t-k \Delta \theta-m \Delta v, t=0
\end{array}\right.
$$

The model is used to represent the evolution process of a system which contains an elastic membrane and plate. The plate has an elastic force and a thermal effect (see [31]). Here $u$ is the vertical deflection of the membrane and $v$ is the vertical deflection of the plate. $\theta$ is the difference of temperature. The coefficients $\rho_{1}, \rho_{2}, \mu, \lambda, m, \tau, \gamma$, and $k$ are nonnegative constants. $\Delta$ denotes the Laplace operator, and $\Delta^{2}$ denotes the biharmonic operator.

In the present paper, we consider the case when $\tau=0$. In this case, Equation (3) can be rewritten as:

$$
\begin{gathered}
\rho_{1} u_{, t t}-\Delta u-\mu \Delta u, t+\lambda \Delta v s .=0, \\
\rho_{2} v_{, t t}+\gamma \Delta^{2} v+\lambda \Delta u-\frac{m^{2}}{k} \Delta v, t=0 .
\end{gathered}
$$

We give the following initial and boundary value conditions:

$$
\left\{\begin{array}{l}
v\left(x_{1}, 0, t\right)=u\left(x_{1}, 0, t\right)=u_{, 2}\left(x_{1}, 0, t\right)=0, x_{1}>0, t>0, \\
v\left(x_{1}, h, t\right)=u\left(x_{1}, h, t\right)=u_{, 2}\left(x_{1}, h, t\right)=0, x_{1}>0, t>0, \\
v\left(0, x_{2}, t\right)=g_{1}\left(x_{2}, t\right), 0 \leq x_{2} \leq h, t>0, \\
u\left(0, x_{2}, t\right)=g_{2}\left(x_{2}, t\right), 0 \leq x_{2} \leq h, t>0, \\
u, 1\left(0, x_{2}, t\right)=g_{3}\left(x_{2}, t\right), 0 \leq x_{2} \leq h, t>0, \\
v\left(x_{1}, x_{2}, 0\right)=u\left(x_{1}, x_{2}, 0\right)=u, t\left(x_{1}, x_{2}, 0\right), 0 \leq x_{2} \leq h, x_{1}>0,
\end{array}\right.
$$

where $g_{i}\left(x_{2}, t\right), i=1,2,3$ are the given functions and meet the following compatibility conditions:

$$
\left\{\begin{array}{l}
g_{1}(0, t)=g_{1}(h, t)=g_{1,2}(0, t)=g_{1,2}(h, t)=0, \\
g_{2}(0, t)=g_{2}(h, t)=g_{2,2}(0, t)=g_{2,2}(h, t)=0, \\
g_{3}(0, t)=g_{3}(h, t)=g_{3,2}(0, t)=g_{3,2}(h, t)=0, \\
g_{1}\left(x_{2}, 0\right)=g_{2}\left(x_{2}, 0\right)=g_{3}\left(x_{2}, 0\right)=0 .
\end{array}\right.
$$

We try to establish the Phragmén-Lindelö $f$ alternative results for the solutions of the biharmonic Equations (4) and (5) under conditions (6) and (7). We firstly define an energy expression of the solutions, then we derive that the energy expression satisfies a second-order differential inequality, and finally we obtain the Phragmén-Lindelöf alternative results of the solutions by solving the second-order inequality. For the inequality is symmetry, we show the application of symmetry in mathematical inequalities in practice. Since the system is a hyperbolic-hyperbolic coupling system, how to define the appropriate energy function will be the greatest innovation in this paper. How to control the energy 
function will be the difficulty of this paper. No similar studies have been found on the spatial properties for the solutions of the biharmonic equations with hyperbolic-hyperbolic coupling equations using the second-order differential inequality. In this paper, we use the comma to represent partial differentiation.,$k$ denotes the differentiation with respect to the direction $x_{k}$, thus $u_{, \alpha}$ denotes $\frac{\partial u}{\partial x_{\alpha}}$, and $u_{, t}$ denotes $\frac{\partial u}{\partial t}$. The usual summation convection is employed with repeated Greek subscripts $\alpha$ summed from 1 to 2 . Hence, $u_{\alpha, \alpha}=\sum_{\alpha=1}^{2} \frac{\partial u_{\alpha}}{\partial x_{\alpha}}$. The symbol $d A=d x_{1} d x_{2}$.

\section{Energy Expression $\Phi(z, t)$}

In order to get the Phragmén-Lindelöf alternative results, we must define an energy expression for the solutions. This expression plays an important role in obtaining our results. The energy expression will be constructed by the following Lemmas.

Lemma 1. Let $u$ and $v$ be classical solutions of problems (4)-(7), we define the a function $\varphi_{1}(z, t)$ as:

$$
\varphi_{1}(z, t)=\frac{\mu}{2} \int_{0}^{t} \int_{L_{z}} \exp (-\omega \eta) u_{, \eta}^{2} \mathrm{~d} x_{2} \mathrm{~d} \eta+\lambda \int_{0}^{t} \int_{L_{z}} \exp (-\omega \eta) u v, \eta \mathrm{d} x_{2} \mathrm{~d} \eta .
$$

$\varphi_{1}(z, t)$ can also be expressed as:

$$
\begin{aligned}
& \varphi_{1}(z, t)=\frac{\omega \rho_{1}}{2} \int_{0}^{t} \int_{0}^{z} \int_{L_{\xi}} \exp (-\omega \eta)(z-\xi) u_{, \eta}^{2} \mathrm{~d} A \mathrm{~d} \eta \\
& +\frac{\rho_{1}}{2} \int_{0}^{z} \int_{L_{\tilde{\xi}}} \exp (-\omega t)(z-\xi) u_{, t}^{2} \mathrm{~d} A \\
& +\frac{\omega}{2} \int_{0}^{t} \int_{0}^{z} \int_{L_{\tilde{\zeta}}} \exp (-\omega \eta)(z-\xi) u_{, \alpha} u_{, \alpha} \mathrm{d} A \mathrm{~d} \eta \\
& +\frac{1}{2} \int_{0}^{z} \int_{L_{\xi}} \exp (-\omega t)(z-\xi) u_{, \alpha} u_{, \alpha} \mathrm{d} A \\
& +\mu \int_{0}^{t} \int_{0}^{z} \int_{L_{\xi}} \exp (-\omega \eta)(z-\xi) u_{, \alpha \eta} u, \alpha \eta \mathrm{d} A \mathrm{~d} \eta \\
& +\lambda \int_{0}^{t} \int_{0}^{z} \int_{L_{\tilde{\xi}}} \exp (-\omega \eta)(z-\xi) u_{, \alpha} v, \alpha \eta \mathrm{d} A \mathrm{~d} \eta \\
& -\int_{0}^{t} \int_{0}^{z} \int_{L_{\tilde{\xi}}} \exp (-\omega \eta) u, \eta u, 1 \mathrm{~d} A \mathrm{~d} \eta \\
& +\lambda \omega \int_{0}^{t} \int_{0}^{z} \int_{L_{\xi}} \exp (-\omega \eta)(z-\xi) u_{, \alpha} v, \alpha \mathrm{d} A \mathrm{~d} \eta \\
& +\lambda \int_{0}^{z} \int_{L_{\xi}} \exp (-\omega t)(z-\xi) u_{, \alpha} v, \alpha \mathrm{d} A \\
& +\lambda \int_{0}^{t} \int_{0}^{z} \int_{L_{\tilde{\xi}}} \exp (-\omega \eta) u_{, 1} v, \eta \mathrm{d} A \mathrm{~d} \eta \\
& +\lambda \omega \int_{0}^{t} \int_{0}^{z} \int_{L_{\tilde{\xi}}} \exp (-\omega \eta) u v, 1 \mathrm{~d} A \mathrm{~d} \eta+\lambda \int_{0}^{z} \int_{L_{\tilde{\xi}}} \exp (-\omega t) u v, 1 \mathrm{~d} A+k_{1}(z, t),
\end{aligned}
$$

where

$$
\begin{aligned}
k_{1}(z, t) & =z \int_{0}^{t} \int_{L_{0}} \exp (-\omega \eta) u_{, \eta} u_{, 1} \mathrm{~d} x_{2} \mathrm{~d} \eta+\frac{\mu}{2} \int_{0}^{t} \int_{L_{0}} \exp (-\omega \eta) u_{, \eta}^{2} \mathrm{~d} x_{2} \mathrm{~d} \eta \\
& +z \mu \int_{0}^{t} \int_{L_{0}} \exp (-\omega \eta) u_{, \eta} u_{, 1 \eta} \mathrm{d} x_{2} \mathrm{~d} \eta+\lambda \int_{0}^{t} \int_{L_{0}} \exp (-\omega \eta) u v_{, \eta} \mathrm{d} x_{2} \mathrm{~d} \eta .
\end{aligned}
$$


Proof. Multiplying both sides of Equation (4) by $\exp (-\omega \eta)(z-\xi) u, \eta$ and integrating, we obtain

$$
\begin{aligned}
& 0=\int_{0}^{t} \int_{0}^{z} \int_{L_{\tilde{\xi}}} \exp (-\omega \eta)(z-\xi) u_{, \eta}\left(\rho_{1} u_{, \eta \eta}-u_{, \alpha \alpha}-\mu u_{, \alpha \alpha \eta}\right. \\
& +\lambda v, \alpha \alpha) \mathrm{d} A \mathrm{~d} \eta \\
& =\frac{\omega \rho_{1}}{2} \int_{0}^{t} \int_{0}^{z} \int_{L_{\xi}} \exp (-\omega \eta)(z-\xi) u_{, \eta}^{2} \mathrm{~d} A \mathrm{~d} \eta \\
& +\frac{\rho_{1}}{2} \int_{0}^{z} \int_{L_{\tilde{\xi}}} \exp (-\omega t)(z-\xi) u_{, t}^{2} \mathrm{~d} A \\
& +\frac{\omega}{2} \int_{0}^{t} \int_{0}^{z} \int_{L_{\tilde{\xi}}} \exp (-\omega \eta)(z-\xi) u_{, \alpha} u, \alpha \mathrm{d} A \mathrm{~d} \eta \\
& +\frac{1}{2} \int_{0}^{z} \int_{L_{\tilde{\xi}}} \exp (-\omega t)(z-\xi) u, \alpha u, \alpha \mathrm{d} A \\
& -\int_{0}^{t} \int_{0}^{z} \int_{L_{\tilde{\xi}}} \exp (-\omega \eta) u_{, \eta} u_{, 1} \mathrm{~d} A \mathrm{~d} \eta \\
& +z \int_{0}^{t} \int_{L_{0}} \exp (-\omega \eta) u_{, \eta} u_{, 1} \mathrm{~d} x_{2} \mathrm{~d} \eta \\
& +\mu \int_{0}^{t} \int_{0}^{z} \int_{L_{\xi}} \exp (-\omega \eta)(z-\xi) u, \alpha \eta u, \alpha \eta \mathrm{d} A \mathrm{~d} \eta \\
& -\frac{\mu}{2} \int_{0}^{t} \int_{L_{z}} \exp (-\omega \eta) u_{, \eta}^{2} \mathrm{~d} x_{2} \mathrm{~d} \eta \\
& +\frac{\mu}{2} \int_{0}^{t} \int_{L_{0}} \exp (-\omega \eta) u_{, \eta}^{2} \mathrm{~d} x_{2} \mathrm{~d} \eta \\
& +z \mu \int_{0}^{t} \int_{L_{0}} \exp (-\omega \eta) u_{, \eta} u_{, 1 \eta} \mathrm{d} x_{2} \mathrm{~d} \eta \\
& +\lambda \int_{0}^{t} \int_{0}^{z} \int_{L_{\xi}} \exp (-\omega \eta)(z-\xi) u_{, \alpha} v, \alpha \eta \mathrm{d} A \mathrm{~d} \eta \\
& +\lambda \omega \int_{0}^{t} \int_{0}^{z} \int_{L_{\xi}} \exp (-\omega \eta)(z-\xi) u_{, \alpha} v, \alpha \mathrm{d} A \mathrm{~d} \eta \\
& +\lambda \int_{0}^{z} \int_{L_{\xi}} \exp (-\omega t)(z-\xi) u_{, \alpha} v, \alpha \mathrm{d} A+\lambda \int_{0}^{t} \int_{0}^{z} \int_{L_{\xi}} \exp (-\omega \eta) u_{, 1} v, \eta \mathrm{d} A \mathrm{~d} \eta \\
& -\lambda \int_{0}^{t} \int_{L_{z}} \exp (-\omega \eta) u v, \eta \mathrm{d} x_{2} \mathrm{~d} \eta+\lambda \int_{0}^{t} \int_{L_{0}} \exp (-\omega \eta) u v, \eta \mathrm{d} x_{2} \mathrm{~d} \eta \\
& +\lambda \omega \int_{0}^{t} \int_{0}^{z} \int_{L_{\xi}} \exp (-\omega \eta) u v_{, 1} \mathrm{~d} A \mathrm{~d} \eta \\
& +\lambda \int_{0}^{z} \int_{L_{\tilde{\zeta}}} \exp (-\omega t) u v_{, 1} \mathrm{~d} A \text {. }
\end{aligned}
$$

By combining Equations (8) and (11), we can get (9). The proof of Lemma 1 is finished.

Lemma 2. We suggest $u$ and $v$ are the classical solutions of problems (4)-(7), and we define a function $\varphi_{2}(z, t)$ as: 


$$
\begin{aligned}
\varphi_{2}(z, t) & =\int_{0}^{t} \int_{0}^{z} \int_{L_{\xi}} \exp (-\omega \eta)(z-\xi)\left(u_{, \alpha \alpha}\right)^{2} \mathrm{~d} A \mathrm{~d} \eta+\mu \omega \int_{0}^{t} \int_{0}^{z} \int_{L_{\xi}} \exp (-\omega \eta)(z-\xi)(u, \alpha \alpha)^{2} \mathrm{~d} A \mathrm{~d} \eta \\
& +\mu \int_{0}^{z} \int_{L_{\xi}} \exp (-\omega \eta)(z-\xi)\left(u_{, \alpha \alpha}\right)^{2} \mathrm{~d} A-\rho_{1} \int_{0}^{t} \int_{0}^{z} \int_{L_{\xi}} \exp (-\omega \eta)(z-\xi) u_{, \beta \eta} u_{, \beta \eta} \mathrm{d} A \mathrm{~d} \eta \\
& +\frac{\rho_{1}}{2} \int_{0}^{t} \int_{0}^{z} \int_{L_{\xi}} \exp (-\omega \eta) u_{, \eta}^{2} \mathrm{~d} A \mathrm{~d} \eta-\omega \rho_{1} \int_{0}^{t} \int_{0}^{z} \int_{L_{\xi}} \exp (-\omega \eta)(z-\xi) u_{, \beta \beta} u_{, \eta} \mathrm{d} A \mathrm{~d} \eta \\
& -\rho_{1} \int_{0}^{z} \int_{L_{\xi}} \exp (-\omega t)(z-\xi) u_{, \beta \beta} u_{,} \mathrm{d} A-\lambda \int_{0}^{t} \int_{0}^{z} \int_{L_{\xi}} \exp (-\omega \eta)(z-\xi) u_{, \beta \beta} v_{, \alpha \alpha} \mathrm{d} A \mathrm{~d} \eta \\
& +k_{2}(z, t),
\end{aligned}
$$

$\varphi_{2}(z, t)$ can also be expressed as:

$$
\varphi_{2}(z, t)=-\frac{\rho_{1}}{2} \int_{0}^{t} \int_{L_{z}} \exp (-\omega \eta) u_{, \eta}^{2} \mathrm{~d} x_{2} \mathrm{~d} \eta
$$

where

$$
k_{2}(z, t)=\frac{\rho_{1} z}{2} \int_{0}^{t} \int_{L_{0}} \exp (-\omega \eta) u_{, \eta}^{2} \mathrm{~d} x_{2} \mathrm{~d} \eta-\rho_{1} z \int_{0}^{t} \int_{L_{0}} \exp (-\omega \eta) u_{, \eta} u_{, 1 \eta} \mathrm{d} x_{2} \mathrm{~d} \eta .
$$

Proof. Multiplying both sides of Equation (4) by $\exp (-\omega \eta)(z-\xi) u_{, \beta \beta}$ and integrating, we can obtain

$$
\begin{aligned}
0 & =\int_{0}^{t} \int_{0}^{z} \int_{L_{\tilde{\xi}}} \exp (-\omega \eta)(z-\xi) u_{, \beta \beta}\left(\rho_{1} u_{, \eta \eta}-u_{, \alpha \alpha}-\mu u_{, \alpha \alpha \eta}+\lambda v_{, \alpha \alpha}\right) \mathrm{d} A \mathrm{~d} \eta \\
& =\rho_{1} \int_{0}^{t} \int_{0}^{z} \int_{L_{\xi}} \exp (-\omega \eta)(z-\xi) u_{, \beta \eta} u_{, \beta \eta} \mathrm{d} A \mathrm{~d} \eta \\
& -\rho_{1} \int_{0}^{t} \int_{0}^{z} \int_{L_{\xi}} \exp (-\omega \eta)(z-\xi) u_{, \eta} u_{, 1 \eta} \mathrm{d} A \mathrm{~d} \eta \\
& +\rho_{1} z \int_{0}^{t} \int_{L_{0}} \exp (-\omega \eta) u_{, \eta} u_{, 1 \eta} \mathrm{d} x_{2} \mathrm{~d} \eta \\
& +\omega \rho_{1} \int_{0}^{t} \int_{0}^{z} \int_{L_{\tilde{\xi}}} \exp (-\omega \eta)(z-\xi) u_{, \beta \beta} u_{, \eta} \mathrm{d} A \mathrm{~d} \eta \\
& +\rho_{1} \int_{0}^{z} \int_{L_{\xi}} \exp (-\omega t)(z-\xi) u_{, \beta \beta} u_{,} \mathrm{d} A \\
& -\int_{0}^{t} \int_{0}^{z} \int_{L_{\tilde{\xi}}} \exp (-\omega \eta)(z-\xi)\left(u_{, \alpha \alpha}\right)^{2} \mathrm{~d} A \mathrm{~d} \eta \\
& -\mu \omega \int_{0}^{t} \int_{0}^{z} \int_{L_{\tilde{\xi}}} \exp (-\omega \eta)(z-\xi)\left(u_{, \alpha \alpha}\right)^{2} \mathrm{~d} A \mathrm{~d} \eta \\
& -\mu \int_{0}^{z} \int_{L_{\tilde{\xi}}} \exp (-\omega t)(z-\xi)\left(u_{, \alpha \alpha}\right)^{2} \mathrm{~d} A \\
& +\lambda \int_{0}^{t} \int_{0}^{z} \int_{L_{\xi}} \exp (-\omega \eta)(z-\xi) u_{, \beta \beta} v_{, \alpha \alpha} \mathrm{d} A \mathrm{~d} \eta
\end{aligned}
$$

By combining Equations (12) and (14), we can get (13).

The proof of Lemma 2 is finished. 
Lemma 3. We suggest $u$ and $v$ are classical solutions of problems (4)-(7), and we define a function $\varphi_{3}(z, t)$ as:

$$
\begin{aligned}
\varphi_{3}(z, t) & =\frac{\omega \rho_{2}}{2} \int_{0}^{t} \int_{0}^{z} \int_{L_{\xi}} \exp (-\omega \eta)(z-\xi) v_{, \eta}^{2} \mathrm{~d} A \mathrm{~d} \eta \\
& +\frac{\rho_{2}}{2} \int_{0}^{z} \int_{L_{\xi}} \exp (-\omega t)(z-\xi) v_{, t}^{2} \mathrm{~d} x_{2} \mathrm{~d} \eta \\
& +\frac{m^{2}}{k} \int_{0}^{t} \int_{0}^{z} \int_{L_{\xi}} \exp (-\omega \eta)(z-\xi) v_{, \alpha \eta} v_{, \alpha \eta} \mathrm{d} A \mathrm{~d} \eta \\
& +\frac{\gamma \omega}{2} \int_{0}^{t} \int_{0}^{z} \int_{L_{\xi}} \exp (-\omega \eta)(z-\xi) v_{, \alpha \beta} v_{, \alpha \beta} \mathrm{d} A \mathrm{~d} \eta \\
& +\frac{\gamma}{2} \int_{0}^{z} \int_{L_{\xi}} \exp (-\omega t) v_{, \alpha \beta} v_{, \alpha \beta} \mathrm{d} A \\
& -\lambda \int_{0}^{t} \int_{0}^{z} \int_{L_{\xi}} \exp (-\omega \eta)(z-\xi) v, \alpha \eta u, \alpha \mathrm{d} A \mathrm{~d} \eta \\
& +\lambda \int_{0}^{t} \int_{0}^{z} \int_{L_{\xi}} \exp (-\omega \eta) v_{, \eta} u_{, 1} \mathrm{~d} A \mathrm{~d} \eta \\
& -2 \gamma \int_{0}^{t} \int_{0}^{z} \int_{L_{\tilde{\zeta}}} \exp (-\omega \eta) v_{, \alpha \eta} v_{, \alpha 1} \mathrm{~d} A \mathrm{~d} \eta \\
& +\frac{2 \gamma \kappa \rho_{2} \omega}{m^{2}} \int_{0}^{t} \int_{0}^{z} \int_{L_{\xi}} \exp (-\omega \eta) v, \eta v, 1 \mathrm{~d} A \mathrm{~d} \eta \\
& +\frac{2 \gamma \kappa \rho_{2}}{m^{2}} \int_{0}^{z} \int_{L_{\xi}} \exp (-\omega \eta) v, t v_{, 1} \mathrm{~d} A \\
& -\frac{2 \kappa \lambda \gamma}{m^{2}} \int_{0}^{t} \int_{0}^{z} \int_{L_{\xi}} \exp (-\omega \eta) u_{, \alpha} v, 1 \alpha \mathrm{d} A \mathrm{~d} \eta+k_{3}(z, t) .
\end{aligned}
$$

$\varphi_{3}(z, t)$ can also be expressed as:

$$
\begin{aligned}
\varphi_{3}(z, t) & =\frac{m^{2}}{2 k} \int_{0}^{t} \int_{L_{z}} \exp (-\omega \eta) v_{, \eta}^{2} \mathrm{~d} x_{2} \mathrm{~d} \eta \\
& +2 \gamma \int_{0}^{t} \int_{L_{z}} \exp (-\omega \eta) v_{, 1 \eta} v_{, 1} \mathrm{~d} x_{2} \mathrm{~d} \eta \\
& -\frac{2 k \lambda \gamma}{m^{2}} \int_{0}^{t} \int_{L_{z}} \exp (-\omega \eta) u_{, 1} v, 11 \mathrm{~d} x_{2} \mathrm{~d} \eta \\
& -\frac{k \gamma^{2}}{m^{2}} \int_{0}^{t} \int_{L_{z}} \exp (-\omega \eta) v_{, \alpha \beta} v_{, \alpha \beta} \mathrm{d} x_{2} \mathrm{~d} \eta \\
& +\frac{2 k \gamma^{2}}{m^{2}} \int_{0}^{t} \int_{L_{z}} \exp (-\omega \eta) v_{, 1 \alpha} v_{, 1 \alpha} \mathrm{d} x_{2} \mathrm{~d} \eta \\
& -\frac{2 k \gamma^{2}}{m^{2}} \int_{0}^{t} \int_{L_{z}} \exp (-\omega \eta) v_{, 1} v_{, 1 \beta \beta} \mathrm{d} x_{2} \mathrm{~d} \eta,
\end{aligned}
$$


with

$$
\begin{aligned}
k_{3}(z, t) & =\left(\frac{m^{2}}{2 k}+\frac{k \rho_{2} \gamma}{m^{2}}\right) \int_{0}^{t} \int_{L_{0}} \exp (-\omega \eta) v_{, \eta}^{2} \mathrm{~d} x_{2} \mathrm{~d} \eta \\
& +\frac{m^{2}}{k} z \int_{0}^{t} \int_{L_{0}} \exp (-\omega \eta) v_{, \eta} v, 1 \eta \mathrm{d} x_{2} \mathrm{~d} \eta \\
& +\gamma z \int_{0}^{t} \int_{L_{0}} \exp (-\omega \eta) v_{, \alpha \eta} v_{, \alpha 1} \mathrm{~d} x_{2} \mathrm{~d} \eta \\
& -\gamma z \int_{0}^{t} \int_{L_{0}} \exp (-\omega \eta) v, \eta v_{1,1 \beta \beta} \mathrm{d} x_{2} \mathrm{~d} \eta \\
& +2 \gamma \int_{0}^{t} \int_{L_{0}} \exp (-\omega \eta) v_{, 1 \eta}{ }_{, 1} \mathrm{~d} x_{2} \mathrm{~d} \eta \\
& -\frac{2 k \lambda \gamma}{m^{2}} \int_{0}^{t} \int_{L_{0}} \exp (-\omega \eta) u_{, 1} v, 11 \mathrm{~d} x_{2} \mathrm{~d} \eta \\
& -\frac{k \gamma^{2}}{m^{2}} \int_{0}^{t} \int_{L_{0}} \exp (-\omega \eta) v_{, \alpha \beta} v_{, \alpha \beta} \mathrm{d} x_{2} \mathrm{~d} \eta \\
& +\frac{2 k \gamma^{2}}{m^{2}} \int_{0}^{t} \int_{L_{0}} \exp (-\omega \eta) v_{, 1 \alpha} v_{, 1 \alpha} \mathrm{d} x_{2} \mathrm{~d} \eta \\
& -\frac{2 k \gamma^{2}}{m^{2}} \int_{0}^{t} \int_{L_{0}} \exp (-\omega \eta) v_{, 1} v_{, 1 \beta \beta} \mathrm{d} x_{2} \mathrm{~d} \eta .
\end{aligned}
$$

Proof. Multiplying both sides of Equation (5) by $\exp (-\omega \eta)(z-\xi) v, \eta$ and integrating, we can obtain

$$
\begin{aligned}
0 & =\int_{0}^{t} \int_{0}^{z} \int_{L_{\tilde{\xi}}} \exp (-\omega \eta)(z-\xi) v_{, \eta}\left(\rho_{2} v_{, \eta \eta}+\gamma v_{, \alpha \alpha \beta \beta}+\lambda u_{, \alpha \alpha}-\frac{m^{2}}{k} v_{, \alpha \alpha \eta}\right) \mathrm{d} A \mathrm{~d} \eta \\
& =\frac{\omega \rho_{2}}{2} \int_{0}^{t} \int_{0}^{z} \int_{L_{\xi}} \exp (-\omega \eta)(z-\xi) v_{, \eta}^{2} \mathrm{~d} A \mathrm{~d} \eta \\
& +\frac{\rho_{2}}{2} \int_{0}^{z} \int_{L_{\tilde{\xi}}} \exp (-\omega t)(z-\xi) v_{, t}^{2} \mathrm{~d} A \\
& -\lambda \int_{0}^{t} \int_{0}^{z} \int_{L_{\tilde{\xi}}} \exp (-\omega \eta)(z-\xi) v_{, \alpha \eta} u_{, \alpha} \mathrm{d} A \mathrm{~d} \eta \\
& +\lambda \int_{0}^{t} \int_{0}^{z} \int_{L_{\xi}} \exp (-\omega \eta) v_{, \eta} u_{, 1} \mathrm{~d} A \mathrm{~d} \eta \\
& +\gamma \int_{0}^{t} \int_{0}^{z} \int_{L_{\tilde{\xi}}} \exp (-\omega \eta)(z-\xi) v_{, \eta} v_{, \alpha \alpha \beta \beta} \mathrm{d} A \mathrm{~d} \eta \\
& +\frac{m^{2}}{k} \int_{0}^{t} \int_{0}^{z} \int_{L_{\tilde{\xi}}} \exp (-\omega \eta)(z-\xi) v_{, \alpha \eta} v_{, \alpha \eta} \mathrm{d} A \mathrm{~d} \eta \\
& -\frac{m^{2}}{2 k} \int_{0}^{t} \int_{L_{z}} \exp (-\omega \eta) v_{, \eta}^{2} \mathrm{~d} x_{2} \mathrm{~d} \eta \\
& +\frac{m^{2}}{2 k} \int_{0}^{t} \int_{L_{0}} \exp (-\omega \eta) v_{, \eta}^{2} \mathrm{~d} x_{2} \mathrm{~d} \eta \\
& +\frac{m^{2}}{k} z \int_{0}^{t} \int_{L_{0}} \exp (-\omega \eta) v_{, \eta} v_{, 1 \eta} \mathrm{d} x_{2} \mathrm{~d} \eta
\end{aligned}
$$


Next, we begin to deal with the term $\gamma \int_{0}^{t} \int_{0}^{z} \int_{L_{\xi}} \exp (-\omega \eta)(z-\xi) v, \eta v, \alpha \alpha \beta \beta \mathrm{d} A \mathrm{~d} \eta$.

$$
\begin{aligned}
& \gamma \int_{0}^{t} \int_{0}^{z} \int_{L_{\xi}} \exp (-\omega \eta)(z-\xi) v_{, \eta} v_{, \alpha \alpha \beta \beta} \mathrm{d} A \mathrm{~d} \eta \\
& =\frac{\gamma \omega}{2} \int_{0}^{t} \int_{0}^{z} \int_{L_{\xi}} \exp (-\omega \eta)(z-\xi) v_{, \alpha \beta} v_{, \alpha \beta} \mathrm{d} A \mathrm{~d} \eta \\
& +\frac{\gamma}{2} \int_{0}^{z} \int_{L_{\xi}} \exp (-\omega \eta)(z-\xi) v_{, \alpha \beta} v_{, \alpha \beta} \mathrm{d} A \\
& -2 \gamma \int_{0}^{t} \int_{0}^{z} \int_{L_{\xi}} \exp (-\omega \eta) v, \alpha \eta v, \alpha 1 \mathrm{~d} A \mathrm{~d} \eta \\
& +\gamma z \int_{0}^{t} \int_{L_{0}} \exp (-\omega \eta) v, \alpha \eta v, \alpha 1 \mathrm{~d} x_{2} \mathrm{~d} \eta \\
& -\gamma z \int_{0}^{t} \int_{L_{0}} \exp (-\omega \eta) v, \eta v_{, 1 \beta \beta} \mathrm{d} x_{2} \mathrm{~d} \eta .
\end{aligned}
$$

Now let us deal with $-2 \gamma \int_{0}^{t} \int_{0}^{z} \int_{L_{\tilde{\zeta}}} \exp (-\omega \eta) v, \alpha \eta v, \alpha 1 \mathrm{~d} A \mathrm{~d} \eta$.

$$
\begin{aligned}
& -2 \gamma \int_{0}^{t} \int_{0}^{z} \int_{L_{\xi}} \exp (-\omega \eta) v_{, \alpha \eta} v_{, \alpha 1} \mathrm{~d} A \mathrm{~d} \eta \\
& =2 \gamma \int_{0}^{t} \int_{0}^{z} \int_{L_{\xi}} \exp (-\omega \eta) v_{, \alpha \alpha \eta} v_{, 1} \mathrm{~d} A \mathrm{~d} \eta \\
& -2 \gamma \int_{0}^{t} \int_{L_{z}} \exp (-\omega \eta) v_{, 1} v_{, 1} \mathrm{~d} x_{2} \mathrm{~d} \eta \\
& +2 \gamma \int_{0}^{t} \int_{L_{0}} \exp (-\omega \eta) v, 1 \eta v_{, 1} \mathrm{~d} x_{2} \mathrm{~d} \eta
\end{aligned}
$$

Using the Equation (5), we can get

$$
\begin{aligned}
& 2 \gamma \int_{0}^{t} \int_{0}^{z} \int_{L_{\tilde{\xi}}} \exp (-\omega \eta) v, \alpha \alpha \eta v_{, 1} \mathrm{~d} A \mathrm{~d} \eta \\
& =2 \gamma \int_{0}^{t} \int_{0}^{z} \int_{L_{\tilde{\xi}}} \exp (-\omega \eta)\left(\frac{k}{m^{2}} \rho_{2} v_{, \eta \eta}+\frac{k}{m^{2}} \gamma v, \alpha \alpha \beta \beta+\frac{k}{m^{2}} \lambda u, \alpha \alpha\right) v_{, 1} \mathrm{~d} A \mathrm{~d} \eta \\
& =-\frac{k \rho_{2} \gamma}{m^{2}} \int_{0}^{t} \int_{L_{z}} \exp (-\omega \eta) v_{, \eta}^{2} \mathrm{~d} x_{2} \mathrm{~d} \eta+\frac{k \rho_{2} \gamma}{m^{2}} \int_{0}^{t} \int_{L_{0}} \exp (-\omega \eta) v_{, \eta}^{2} \mathrm{~d} x_{2} \mathrm{~d} \eta \\
& +\frac{2 k \gamma \rho_{2} \omega}{m^{2}} \int_{0}^{t} \int_{0}^{z} \int_{L_{\tilde{\xi}}} \exp (-\omega \eta) v_{, \eta} v_{, 1} \mathrm{~d} A \mathrm{~d} \eta+\frac{2 k \gamma \rho_{2}}{m^{2}} \int_{0}^{z} \int_{L_{\tilde{\xi}}} \exp (-\omega \eta) v_{,} v, 1 \mathrm{~d} A \\
& +\frac{2 k \gamma^{2}}{m^{2}} \int_{0}^{t} \int_{0}^{z} \int_{L_{\tilde{\xi}}} \exp (-\omega \eta) v_{, 1} v_{, \alpha \alpha \beta \beta} \mathrm{d} A \mathrm{~d} \eta \\
& -\frac{2 k \lambda \gamma}{m^{2}} \int_{0}^{t} \int_{0}^{z} \int_{L_{\tilde{\xi}}} \exp (-\omega \eta) u_{, \alpha} v_{, 1 \alpha} \mathrm{d} A \mathrm{~d} \eta \\
& +\frac{2 k \lambda \gamma}{m^{2}} \int_{0}^{t} \int_{L_{z}} \exp (-\omega \eta) u_{, 1} v_{, 11} \mathrm{~d} x_{2} \mathrm{~d} \eta \\
& -\frac{2 k \lambda \gamma}{m^{2}} \int_{0}^{t} \int_{L_{0}} \exp (-\omega \eta) u_{, 1} v_{, 11} \mathrm{~d} x_{2} \mathrm{~d} \eta .
\end{aligned}
$$

Now, let us deal with the term $\frac{2 k \gamma^{2}}{m^{2}} \int_{0}^{t} \int_{0}^{z} \int_{L_{\tilde{\xi}}} \exp (-\omega \eta) v, 1 v, \alpha \alpha \beta \beta \mathrm{d} A \mathrm{~d} \eta$. 


$$
\begin{aligned}
& \frac{2 k \gamma^{2}}{m^{2}} \int_{0}^{t} \int_{0}^{z} \int_{L_{\xi}} \exp (-\omega \eta) v_{, 1} v, \alpha \alpha \beta \beta \\
& =-\frac{2 k \gamma^{2}}{m^{2}} \int_{0}^{t} \int_{0}^{z} \int_{L_{\xi}} \exp (-\omega \eta) v_{, 1 \alpha} v_{, \alpha \beta \beta} \mathrm{d} A \mathrm{~d} \eta+\frac{2 k \gamma^{2}}{m^{2}} \int_{0}^{t} \int_{L_{z}} \exp (-\omega \eta) v_{, 1} v_{, 1 \beta \beta} \mathrm{d} x_{2} \mathrm{~d} \eta \\
& -\frac{2 k \gamma^{2}}{m^{2}} \int_{0}^{t} \int_{L_{0}} \exp (-\omega \eta) v_{, 1} v_{, 1 \beta \beta} \mathrm{d} x_{2} \mathrm{~d} \eta \\
& =\frac{k \gamma^{2}}{m^{2}} \int_{0}^{t} \int_{L_{z}} \exp (-\omega \eta) v_{, \alpha \beta} v_{, \alpha \beta} \mathrm{d} x_{2} \mathrm{~d} \eta-\frac{k \gamma^{2}}{m^{2}} \int_{0}^{t} \int_{L_{0}} \exp (-\omega \eta) v_{, \alpha \beta} v_{, \alpha \beta} \mathrm{d} x_{2} \mathrm{~d} \eta \\
& -\frac{2 k \gamma^{2}}{m^{2}} \int_{0}^{t} \int_{L_{z}} \exp (-\omega \eta) v_{, 1 \alpha} v_{, 1 \alpha} \mathrm{d} x_{2} \mathrm{~d} \eta+\frac{2 k \gamma^{2}}{m^{2}} \int_{0}^{t} \int_{L_{0}} \exp (-\omega \eta) v_{, 1 \alpha} v_{, 1 \alpha} \mathrm{d} x_{2} \mathrm{~d} \eta \\
& +\frac{2 k \gamma^{2}}{m^{2}} \int_{0}^{t} \int_{L_{z}} \exp (-\omega \eta) v_{, 1} v_{, 1 \beta \beta} \mathrm{d} x_{2} \mathrm{~d} \eta-\frac{2 k \gamma^{2}}{m^{2}} \int_{0}^{t} \int_{L_{0}} \exp (-\omega \eta) v_{, 1} v_{, 1 \beta \beta} \mathrm{d} x_{2} \mathrm{~d} \eta
\end{aligned}
$$

A combination of (15) and (17)-(21), we obtain

$$
\begin{aligned}
\varphi_{3}(z, t) & =\frac{m^{2}}{2 k} \int_{0}^{t} \int_{L_{z}} \exp (-\omega \eta) v_{, \eta}^{2} \mathrm{~d} x_{2} \mathrm{~d} \eta+2 \gamma \int_{0}^{t} \int_{L_{z}} \exp (-\omega \eta) v_{, 1 \eta} v_{, 1} \mathrm{~d} x_{2} \mathrm{~d} \eta \\
& -\frac{2 k \lambda \gamma}{m^{2}} \int_{0}^{t} \int_{L_{z}} \exp (-\omega \eta) u_{, 1} v_{, 11} \mathrm{~d} x_{2} \mathrm{~d} \eta-\frac{k \gamma^{2}}{m^{2}} \int_{0}^{t} \int_{L_{z}} \exp (-\omega \eta) v_{, \alpha \beta} v_{, \alpha \beta} \mathrm{d} x_{2} \mathrm{~d} \eta \\
& +\frac{2 k \gamma^{2}}{m^{2}} \int_{0}^{t} \int_{L_{z}} \exp (-\omega \eta) v_{, 1 \alpha} v_{, 1 \alpha} \mathrm{d} x_{2} \mathrm{~d} \eta-\frac{2 k \gamma^{2}}{m^{2}} \int_{0}^{t} \int_{L_{z}} \exp (-\omega \eta) v_{, 1} v_{, 1 \beta \beta} \mathrm{d} x_{2} \mathrm{~d} \eta
\end{aligned}
$$

The proof of Lemma 3 is finished.

Lemma 4. We suggest $u$ and $v$ are classical solutions of problems (4)-(7), and we define a function $\varphi_{4}(z, t)$ as :

$$
\begin{aligned}
\varphi_{4}(z, t) & =-\frac{\rho_{2}}{2} \int_{0}^{t} \int_{L_{z}} \exp (-\omega \eta) v_{, \eta}^{2} \mathrm{~d} x_{2} \mathrm{~d} \eta+\gamma \int_{0}^{t} \int_{L_{z}} \exp (-\omega \eta) v_{, 1 \alpha \beta} v_{, \alpha \beta} \mathrm{d} x_{2} \mathrm{~d} \eta \\
& -\frac{\gamma}{2} \int_{0}^{t} \int_{L_{z}} \exp (-\omega \eta) v_{, 1 \alpha} v_{, 1 \alpha} \mathrm{d} x_{2} \mathrm{~d} \eta+\frac{\gamma}{2} \int_{0}^{t} \int_{L_{z}} \exp (-\omega \eta) v_{, 11}^{2} \mathrm{~d} x_{2} \mathrm{~d} \eta \\
& -\frac{\gamma}{2} \int_{0}^{t} \int_{L_{z}} \exp (-\omega \eta) v_{, 12}^{2} \mathrm{~d} x_{2} \mathrm{~d} \eta .
\end{aligned}
$$

Then, $\varphi_{4}(z, t)$ can also be expressed as:

$$
\begin{aligned}
\varphi_{4}(z, t) & =-\rho_{2} \int_{0}^{t} \int_{0}^{z} \int_{L_{\xi}} \exp (-\omega \eta)(z-\xi) v_{, 1 \eta}^{2} \mathrm{~d} A \mathrm{~d} \eta+\frac{m^{2}}{2 k} \int_{0}^{z} \int_{L_{\xi}} \exp (-\omega t)(z-\xi) v_{, 1 \alpha} v_{, 1 \alpha} \mathrm{d} A \\
& +\gamma \int_{0}^{t} \int_{0}^{z} \int_{L_{\xi}} \exp (-\omega \eta) v_{, 1 \alpha \beta} v_{1 \alpha \beta} \mathrm{d} A \mathrm{~d} \eta-\rho_{2} \omega \int_{0}^{t} \int_{0}^{z} \int_{L_{\xi}} \exp (-\omega \eta)(z-\xi) v_{, 11} v_{, \eta} \mathrm{d} A \mathrm{~d} \eta \\
& -\rho_{2} \int_{0}^{z} \int_{L_{\tilde{\zeta}}} \exp (-\omega t)(z-\xi) v_{, 11} v_{, t} \mathrm{~d} A+\lambda \int_{0}^{t} \int_{0}^{z} \int_{L_{\xi}} \exp (-\omega \eta)(z-\xi) v_{, 11 \alpha} u_{, \alpha} \mathrm{d} A \mathrm{~d} \eta \\
& -\lambda \int_{0}^{t} \int_{0}^{z} \int_{L_{\xi}} \exp (-\omega \eta) v_{, 11} u_{, 1} \mathrm{~d} A \mathrm{~d} \eta+\int_{0}^{t} \int_{0}^{z} \int_{L_{\xi}} \exp (-\omega \eta) v_{, 12} v_{, 2 \eta} \mathrm{d} A \mathrm{~d} \eta+k_{4}(z, t),
\end{aligned}
$$


with

$$
\begin{aligned}
& k_{4}(z, t)=-\frac{\rho_{2}}{2} \int_{0}^{t} \int_{L_{0}} \exp (-\omega \eta) v_{, \eta}^{2} \mathrm{~d} x_{2} \mathrm{~d} \eta \\
&-\rho_{2} z \int_{0}^{z} \int_{L_{0}} \exp (-\omega \eta) v_{, 1 \eta} v, \eta \mathrm{d} x_{2} \mathrm{~d} \eta \\
&+\frac{m^{2}}{k} z \int_{0}^{t} \int_{L_{0}} \exp (-\omega \eta) v_{, 12} v, 2 \eta \mathrm{d} x_{2} \mathrm{~d} \eta \\
&+\gamma \int_{0}^{t} \int_{L_{0}} \exp (-\omega \eta) v, 1 \alpha \beta,, \alpha \beta \\
& \mathrm{d} x_{2} \mathrm{~d} \eta \\
&-\frac{\gamma}{2} \int_{0}^{t} \int_{L_{0}} \exp (-\omega \eta) v_{, 1 \alpha} v_{, 1 \alpha} \mathrm{d} x_{2} \mathrm{~d} \eta \\
&-\gamma z \int_{0}^{t} \int_{L_{0}} \exp (-\omega \eta) v_{, 11 \alpha} v, \alpha 1 \mathrm{~d} x_{2} \mathrm{~d} \eta \\
&+\frac{\gamma}{2} \int_{0}^{t} \int_{L_{0}} \exp (-\omega \eta) v_{, 11}^{2} \mathrm{~d} x_{2} \mathrm{~d} \eta \\
&-\frac{\gamma}{2} \int_{0}^{t} \int_{L_{0}} \exp (-\omega \eta) v_{, 12}^{2} \mathrm{~d} x_{2} \mathrm{~d} \eta \\
&+\gamma z \int_{0}^{t} \int_{L_{0}} \exp (-\omega \eta) v_{, 11} v, 1 \beta \beta \mathrm{d} x_{2} \mathrm{~d} \eta \\
&+\lambda z \int_{0}^{t} \int_{L_{0}} \exp (-\omega \eta) v_{, 11} u_{, 1} \mathrm{~d} x_{2} \mathrm{~d} \eta .
\end{aligned}
$$

Proof. Multiplying both sides of Equation (5) by $\exp (-\omega \eta)(z-\xi) v, 11$ and integrating, we can obtain

$$
\begin{aligned}
0 & =\rho_{2} \int_{0}^{t} \int_{0}^{z} \int_{L_{\tilde{\xi}}} \exp (-\omega \eta)(z-\xi) v, 11 v, \eta \eta \mathrm{d} A \mathrm{~d} \eta \\
& +\gamma \int_{0}^{t} \int_{0}^{z} \int_{L_{\tilde{\xi}}} \exp (-\omega \eta)(z-\xi) v_{, 11} v, \alpha \alpha \beta \beta \\
& +\lambda \int_{0}^{t} \int_{0}^{z} \int_{L_{\tilde{\xi}}} \exp (-\omega \eta)(z-\xi) v_{, 11} u_{, \alpha \alpha} \mathrm{d} A \mathrm{~d} \eta \\
& -\frac{m^{2}}{k} \int_{0}^{t} \int_{0}^{z} \int_{L_{\tilde{\xi}}} \exp (-\omega \eta)(z-\xi) v_{, 11} v, \alpha \alpha \eta \mathrm{d} A \mathrm{~d} \eta .
\end{aligned}
$$

Using the divergence theorem, the first term on the right of Equation (22) can be rewritten as

$$
\begin{aligned}
& \rho_{2} \int_{0}^{t} \int_{0}^{z} \int_{L_{\xi}} \exp (-\omega \eta)(z-\xi) v_{, 11} v_{, \eta \eta} \mathrm{d} A \mathrm{~d} \eta \\
& =\rho_{2} \int_{0}^{t} \int_{0}^{z} \int_{L_{\xi}} \exp (-\omega \eta)(z-\xi) v_{, 1 \eta} v_{, 1 \eta} \mathrm{d} A \mathrm{~d} \eta-\frac{\rho_{2}}{2} \int_{0}^{t} \int_{L_{z}} \exp (-\omega \eta) v_{, \eta}^{2} \mathrm{~d} x_{2} \mathrm{~d} \eta \\
& +\frac{\rho_{2}}{2} \int_{0}^{t} \int_{L_{0}} \exp (-\omega \eta) v_{, \eta}^{2} \mathrm{~d} x_{2} \mathrm{~d} \eta+\rho_{2} z \int_{0}^{t} \int_{L_{0}} \exp (-\omega \eta) v_{, 1 \eta} v_{, \eta} \mathrm{d} x_{2} \mathrm{~d} \eta \\
& +\rho_{2} \omega \int_{0}^{t} \int_{0}^{z} \int_{L_{\xi}} \exp (-\omega \eta)(z-\xi) v_{, 11} v_{, \eta} \mathrm{d} A \mathrm{~d} \eta \\
& +\rho_{2} \int_{0}^{z} \int_{L_{\tilde{\xi}}} \exp (-\omega t)(z-\xi) v_{, 11} v_{, t} \mathrm{~d} A .
\end{aligned}
$$


Similarly, the second term on the right of Equation (22) can be rewritten as

$\gamma \int_{0}^{t} \int_{0}^{z} \int_{L_{\tilde{\zeta}}} \exp (-\omega \eta)(z-\xi) v_{, 11} v_{, \alpha \alpha \beta \beta} \mathrm{d} A \mathrm{~d} \eta$

$=-\gamma \int_{0}^{t} \int_{0}^{z} \int_{L_{\xi}} \exp (-\omega \eta) v, 1 \alpha \beta^{v}, 1 \alpha \beta \mathrm{d} A \mathrm{~d} \eta+\gamma \int_{0}^{t} \int_{L_{z}} \exp (-\omega \eta) v, 1 \alpha \beta^{v}, \alpha \beta \mathrm{d} x_{2} \mathrm{~d} \eta$

$-\gamma \int_{0}^{t} \int_{L_{0}} \exp (-\omega \eta) v_{, 1 \alpha \beta}{ }_{, \alpha \beta} \mathrm{d} x_{2} \mathrm{~d} \eta-\frac{\gamma}{2} \int_{0}^{t} \int_{L_{z}} \exp (-\omega \eta) v_{, 1 \alpha} v_{, 1 \alpha} \mathrm{d} x_{2} \mathrm{~d} \eta$

$+\frac{\gamma}{2} \int_{0}^{t} \int_{L_{0}} \exp (-\omega \eta) v_{, 1 \alpha} v, 1 \alpha \mathrm{d} x_{2} \mathrm{~d} \eta+\gamma z \int_{0}^{t} \int_{L_{0}} \exp (-\omega \eta) v_{, 11 \alpha} v, \alpha 1 \mathrm{~d} x_{2} \mathrm{~d} \eta$

$+\frac{\gamma}{2} \int_{0}^{t} \int_{L_{z}} \exp (-\omega \eta) v_{, 11}^{2} \mathrm{~d} x_{2} \mathrm{~d} \eta-\frac{\gamma}{2} \int_{0}^{t} \int_{L_{0}} \exp (-\omega \eta) v_{, 11}^{2} \mathrm{~d} x_{2} \mathrm{~d} \eta$

$-\frac{\gamma}{2} \int_{0}^{t} \int_{L_{z}} \exp (-\omega \eta) v_{, 12}^{2} \mathrm{~d} x_{2} \mathrm{~d} \eta+\frac{\gamma}{2} \int_{0}^{t} \int_{L_{0}} \exp (-\omega \eta) v_{, 12}^{2} \mathrm{~d} x_{2} \mathrm{~d} \eta$

$-\gamma z \int_{0}^{t} \int_{L_{0}} \exp (-\omega \eta) v, 11 v, 1 \beta \beta \mathrm{d} x_{2} \mathrm{~d} \eta$.

The third term on the right of Equation (22) can be rewritten as

$$
\begin{aligned}
& \lambda \int_{0}^{t} \int_{0}^{z} \int_{L_{\xi}} \exp (-\omega \eta)(z-\xi) v_{, 11} u, \alpha \alpha \\
& \mathrm{d} A \mathrm{~d} \eta \\
& =-\lambda \int_{0}^{t} \int_{0}^{z} \int_{L_{\xi}} \exp (-\omega \eta)(z-\xi) v_{, 11 \alpha} u, \alpha \mathrm{d} A \mathrm{~d} \eta \\
& +\lambda \int_{0}^{t} \int_{0}^{z} \int_{L_{\xi}} \exp (-\omega \eta) v_{, 11} u_{, 1} \mathrm{~d} A \mathrm{~d} \eta \\
& -\lambda z \int_{0}^{t} \int_{L_{0}} \exp (-\omega \eta) v_{, 11} u_{, 1} \mathrm{~d} x_{2} \mathrm{~d} \eta .
\end{aligned}
$$

The fourth term on the right of Equation (22) can be rewritten as

$$
\begin{aligned}
& -\frac{m^{2}}{k} \int_{0}^{t} \int_{0}^{z} \int_{L_{\xi}} \exp (-\omega \eta)(z-\xi) v, 11 v, \alpha \alpha \eta \mathrm{d} A \mathrm{~d} \eta \\
& =-\frac{m^{2}}{2 k} \int_{0}^{z} \int_{L_{\xi}} \exp (-\omega t)(z-\xi) v_{, 1 \alpha} v, 1 \alpha \mathrm{d} A \\
& -\frac{m^{2}}{k} z \int_{0}^{t} \int_{L_{0}} \exp (-\omega \eta)(z-\xi) v_{, 12} v, 2 \eta \mathrm{d} x_{2} \mathrm{~d} \eta \\
& -\int_{0}^{t} \int_{0}^{z} \int_{L_{\xi}} \exp (-\omega \eta) v_{, 12} v_{, 2 \eta} \mathrm{d} A \mathrm{~d} \eta .
\end{aligned}
$$

A combination of (24)-(28) gives (23). The proof of Lemma 4 is finished.

Lemma 5. We define new energy expressions $\varphi(z, t)$ and $\Phi(z, t)$ as follows:

$$
\varphi(z, t)=\varphi_{1}(z, t)+k_{1} \varphi_{2}(z, t)+\varphi_{3}(z, t)+k_{2} \varphi_{4}(z, t),
$$

and

$$
\Phi(z, t)=\int_{0}^{t} \varphi(z, s) d s
$$

The following second-order partial differential inequality holds

$$
|\Phi(z, t)| \leq k_{3} \frac{\partial^{2} \Phi(z, t)}{\partial z^{2}}
$$


where $k_{1} k_{2}$ and $k_{3}$ are positive constance to be defined later.

Proof. From (9), we can get

$$
\begin{aligned}
& \frac{\partial^{2} \varphi_{1}(z, t)}{\partial z^{2}}=\frac{\omega \rho_{1}}{2} \int_{0}^{t} \int_{L_{z}} \exp (-\omega \eta) u_{, \eta}^{2} \mathrm{~d} x_{2} \mathrm{~d} \eta \\
& +\frac{\rho_{1}}{2} \int_{L_{z}} \exp (-\omega t) u_{, t}^{2} \mathrm{~d} x_{2} \\
& +\frac{\omega}{2} \int_{0}^{t} \int_{L_{z}} \exp (-\omega \eta) u_{, \alpha} u_{, \alpha} \mathrm{d} x_{2} \mathrm{~d} \eta \\
& +\frac{1}{2} \int_{L_{z}} \exp (-\omega t) u_{, \alpha} u_{, \alpha} \mathrm{d} x_{2} \\
& -\lambda \int_{0}^{t} \int_{L_{z}} \exp (-\omega \eta) u_{, 1 \eta} u_{, 1} \mathrm{~d} x_{2} \mathrm{~d} \eta \\
& -\lambda \int_{0}^{t} \int_{L_{z}} \exp (-\omega \eta) u_{, \eta} u_{, 11} \mathrm{~d} x_{2} \mathrm{~d} \eta \\
& +\lambda \int_{0}^{t} \int_{L_{z}} \exp (-\omega \eta) u_{, 11} v, \eta \mathrm{d} x_{2} \mathrm{~d} \eta \\
& +\lambda \int_{0}^{t} \int_{L_{z}} \exp (-\omega \eta) u_{1} v_{, 1 \eta} \mathrm{d} x_{2} \mathrm{~d} \eta \\
& +\lambda \omega \int_{0}^{t} \int_{L_{z}} \exp (-\omega \eta) u_{, 1}{ }_{, 1} \mathrm{~d} x_{2} \mathrm{~d} \eta \\
& +\lambda \omega \int_{0}^{t} \int_{L_{z}} \exp (-\omega \eta) u v,{ }_{11} \mathrm{~d} x_{2} \mathrm{~d} \eta \\
& +\mu \int_{0}^{t} \int_{L_{z}} \exp (-\omega \eta) u_{, \alpha \eta} u_{, \alpha \eta} \mathrm{d} x_{2} \mathrm{~d} \eta \\
& +\lambda \int_{0}^{t} \int_{L_{z}} \exp (-\omega \eta) u_{, \alpha} v, \alpha \eta \mathrm{d} x_{2} \mathrm{~d} \eta \\
& +\lambda \int_{L_{z}} \exp (-\omega t) u_{, 1} v, 1 \mathrm{~d} x_{2} \\
& +\lambda \int_{L_{z}} \exp (-\omega t) u v, 11 \mathrm{~d} x_{2} \\
& +\lambda \omega \int_{0}^{t} \int_{L_{z}} \exp (-\omega \eta) u_{, \alpha} v_{, \alpha} \mathrm{d} x_{2} \mathrm{~d} \eta \\
& +\lambda \int_{L_{z}} \exp (-\omega t) u_{, \alpha} v_{, \alpha} \mathrm{d} x_{2} \text {. }
\end{aligned}
$$

From (12), we can get

$$
\begin{aligned}
\frac{\partial^{2} \varphi_{2}(z, t)}{\partial z^{2}} & =\int_{0}^{t} \int_{L_{z}} \exp (-\omega \eta)\left(u_{, \alpha \alpha}\right)^{2} \mathrm{~d} x_{2} \mathrm{~d} \eta \\
& +\mu \omega \int_{0}^{t} \int_{L_{z}} \exp (-\omega \eta)\left(u_{, \alpha \alpha}\right)^{2} \mathrm{~d} x_{2} \mathrm{~d} \eta \\
& +\mu \int_{L_{z}} \exp (-\omega \eta)\left(u_{, \alpha \alpha}\right)^{2} \mathrm{~d} x_{2} \\
& -\rho_{1} \int_{0}^{t} \int_{L_{z}} \exp (-\omega \eta) u_{, \beta \eta} u_{, \beta \eta} \mathrm{d} x_{2} \mathrm{~d} \eta \\
& +\rho_{1} \int_{0}^{t} \int_{L_{z}} \exp (-\omega \eta) u_{, 1 \eta} u_{, 1} \mathrm{~d} x_{2} \mathrm{~d} \eta .
\end{aligned}
$$

From (15), we can get 


$$
\begin{aligned}
\frac{\partial^{2} \varphi_{3}(z, t)}{\partial z^{2}} & =\frac{\omega \rho_{2}}{2} \int_{0}^{t} \int_{L_{z}} \exp (-\omega \eta) v_{, \eta}^{2} \mathrm{~d} x_{2} \mathrm{~d} \eta+\frac{\rho_{2}}{2} \int_{L_{z}} \exp (-\omega t) v_{, t}^{2} \mathrm{~d} x_{2} \\
& +\frac{m^{2}}{k} \int_{0}^{t} \int_{L_{z}} \exp (-\omega \eta) v_{, \alpha \eta} v_{, \alpha \eta} \mathrm{d} x_{2} \mathrm{~d} \eta+\frac{\gamma \omega}{2} \int_{0}^{t} \int_{L_{z}} \exp (-\omega \eta) v_{, \alpha \beta} v_{, \alpha \beta} \mathrm{d} x_{2} \mathrm{~d} \eta \\
& +\frac{\gamma}{2} \int_{L_{z}} \exp (-\omega t) v_{, \alpha \beta} v_{, \alpha \beta} \mathrm{d} x_{2}-\lambda \int_{0}^{t} \int_{L_{z}} \exp (-\omega \eta) v_{, \alpha \eta} u_{, \alpha} \mathrm{d} x_{2} \mathrm{~d} \eta \\
& +\lambda \int_{0}^{t} \int_{L_{z}} \exp (-\omega \eta) v_{, 1 \eta} u, 1 \mathrm{~d} x_{2} \mathrm{~d} \eta+\lambda \int_{0}^{t} \int_{L_{z}} \exp (-\omega \eta) v_{, \eta} u_{, 11} \mathrm{~d} x_{2} \mathrm{~d} \eta \\
& +\frac{2 \gamma \kappa \rho_{2} \omega}{m^{2}} \int_{0}^{t} \int_{L_{z}} \exp (-\omega \eta) v_{, 1 \eta} v, 1 \mathrm{~d} x_{2} \mathrm{~d} \eta+\frac{2 \gamma \kappa \rho_{2} \omega}{m^{2}} \int_{0}^{t} \int_{L_{z}} \exp (-\omega \eta) v_{, \eta} v_{, 11} \mathrm{~d} x_{2} \mathrm{~d} \eta \\
& +\frac{2 \gamma \kappa \rho_{2}}{m^{2}} \int_{L_{z}} \exp (-\omega t) v, 1 t v_{, 1} \mathrm{~d} x_{2}+\frac{2 \gamma \kappa \rho_{2}}{m^{2}} \int_{L_{z}} \exp (-\omega t) v_{, t} v_{, 11} \mathrm{~d} x_{2} \\
& -\frac{2 \kappa \lambda \gamma}{m^{2}} \int_{0}^{t} \int_{L_{z}} \exp (-\omega \eta) u_{, 1 \alpha} v, 1 \alpha \mathrm{d} x_{2} \mathrm{~d} \eta-\frac{2 \kappa \lambda \gamma}{m^{2}} \int_{0}^{t} \int_{L_{z}} \exp (-\omega \eta) u_{, \alpha} v_{, 11 \alpha} \mathrm{d} x_{2} \mathrm{~d} \eta .
\end{aligned}
$$

From (23), we can get

$$
\begin{aligned}
\frac{\partial^{2} \varphi_{4}(z, t)}{\partial z^{2}} & =-\rho_{2} \int_{0}^{t} \int_{L_{z}} \exp (-\omega \eta) v_{, 1 \eta}^{2} \mathrm{~d} x_{2} \mathrm{~d} \eta+\frac{m^{2}}{2 k} \int_{L_{z}} \exp (-\omega t) v_{, 1 \alpha} v_{, 1 \alpha} \mathrm{d} x_{2} \\
& +\gamma \int_{0}^{t} \int_{L_{z}} \exp (-\omega \eta) v_{11 \alpha \beta} v_{, 1 \alpha \beta} \mathrm{d} x_{2} \mathrm{~d} \eta-\rho_{2} \omega \int_{0}^{t} \int_{L_{z}} \exp (-\omega \eta) v_{, 11} v_{, \eta} \mathrm{d} x_{2} \mathrm{~d} \eta \\
& +\rho_{2} \int_{L_{z}} \exp (-\omega \eta) v_{, 11} v, t \mathrm{~d} x_{2}+\lambda \int_{0}^{t} \int_{L_{z}} \exp (-\omega \eta) v_{, 11 \alpha} u_{, \alpha} \mathrm{d} x_{2} \mathrm{~d} \eta \\
& -\lambda \int_{0}^{t} \int_{L_{z}} \exp (-\omega \eta) v, 111 u_{1} \mathrm{~d} x_{2} \mathrm{~d} \eta-\lambda \int_{0}^{t} \int_{L_{z}} \exp (-\omega \eta) v_{, 11} u_{, 11} \mathrm{~d} x_{2} \mathrm{~d} \eta \\
& +\int_{0}^{t} \int_{L_{z}} \exp (-\omega \eta) v_{, 112} v_{, 2 \eta} \mathrm{d} x_{2} \mathrm{~d} \eta+\int_{0}^{t} \int_{L_{z}} \exp (-\omega \eta) v_{, 12} v_{12 \eta} \mathrm{d} x_{2} \mathrm{~d} \eta
\end{aligned}
$$

A combination of (8), (13), (21), (22) and (29) leads to

$$
\begin{aligned}
\varphi(z, t) & =\frac{\mu}{2} \int_{0}^{t} \int_{L_{z}} \exp (-\omega \eta) u_{, \eta}^{2} \mathrm{~d} x_{2} \mathrm{~d} \eta+\lambda \int_{0}^{t} \int_{L_{z}} \exp (-\omega \eta) u v, \eta \mathrm{d} x_{2} \mathrm{~d} \eta \\
& -k_{1} \frac{\rho_{1}}{2} \int_{0}^{t} \int_{L_{z}} \exp (-\omega \eta) u_{, \eta}^{2} \mathrm{~d} x_{2} \mathrm{~d} \eta-k_{2} \frac{\gamma}{2} \int_{0}^{t} \int_{L_{z}} \exp (-\omega \eta) v_{, 12}^{2} \mathrm{~d} x_{2} \mathrm{~d} \eta \\
& +\frac{m^{2}}{2 k} \int_{0}^{t} \int_{L_{z}} \exp (-\omega \eta) v_{, \eta}^{2} \mathrm{~d} x_{2} \mathrm{~d} \eta+2 \gamma \int_{0}^{t} \int_{L_{z}} \exp (-\omega \eta) v_{, 1 \eta} v_{, 1} \mathrm{~d} x_{2} \mathrm{~d} \eta \\
& -\frac{2 k \lambda \gamma}{m^{2}} \int_{0}^{t} \int_{L_{z}} \exp (-\omega \eta) u_{, 1} v, 11 \mathrm{~d} x_{2} \mathrm{~d} \eta-\frac{k \gamma^{2}}{m^{2}} \int_{0}^{t} \int_{L_{z}} \exp (-\omega \eta) v_{, \alpha \beta} v_{, \alpha \beta} \mathrm{d} x_{2} \mathrm{~d} \eta \\
& +\frac{2 k \gamma^{2}}{m^{2}} \int_{0}^{t} \int_{L_{z}} \exp (-\omega \eta) v_{, 1 \alpha} v_{, 1 \alpha} \mathrm{d} x_{2} \mathrm{~d} \eta-\frac{2 k \gamma^{2}}{m^{2}} \int_{0}^{t} \int_{L_{z}} \exp (-\omega \eta) v_{, 1} v_{, 1 \beta \beta} \mathrm{d} x_{2} \mathrm{~d} \eta \\
& -k_{2} \frac{\rho_{2}}{2} \int_{0}^{t} \int_{L_{z}} \exp (-\omega \eta) v_{, \eta}^{2} \mathrm{~d} x_{2} \mathrm{~d} \eta+k_{2} \gamma \int_{0}^{t} \int_{L_{z}} \exp (-\omega \eta) v_{, 1 \alpha \beta} v_{, \alpha \beta} \mathrm{d} x_{2} \mathrm{~d} \eta \\
& -k_{2} \frac{\gamma}{2} \int_{0}^{t} \int_{L_{z}} \exp (-\omega \eta) v_{, 1 \alpha} v_{, 1 \alpha} \mathrm{d} x_{2} \mathrm{~d} \eta+k_{2} \frac{\gamma}{2} \int_{0}^{t} \int_{L_{z}} \exp (-\omega \eta) v_{, 11}^{2} \mathrm{~d} x_{2} \mathrm{~d} \eta .
\end{aligned}
$$

Combining (32)-(36), we have 


$$
\begin{aligned}
& \frac{\partial^{2} \varphi(z, t)}{\partial z^{2}}=\frac{\omega \rho_{1}}{2} \int_{0}^{t} \int_{L_{z}} \exp (-\omega \eta) u_{, \eta}^{2} \mathrm{~d} x_{2} \mathrm{~d} \eta+\frac{\rho_{1}}{2} \int_{L_{z}} \exp (-\omega t) u_{, t}^{2} \mathrm{~d} x_{2} \\
& +\frac{\omega}{2} \int_{0}^{t} \int_{L_{z}} \exp (-\omega \eta) u_{, \alpha} u_{, \alpha} \mathrm{d} x_{2} \mathrm{~d} \eta+\frac{1}{2} \int_{L_{z}} \exp (-\omega t) u_{, \alpha} u_{, \alpha} \mathrm{d} x_{2} \\
& +u \int_{0}^{t} \int_{L_{z}} \exp (-\omega \eta) u_{, \alpha \eta} u_{, \alpha \eta} \mathrm{d} x_{2} \mathrm{~d} \eta+\lambda \omega \int_{0}^{t} \int_{L_{z}} \exp (-\omega \eta) u_{, \alpha} v, \alpha \mathrm{d} x_{2} \mathrm{~d} \eta \\
& +\lambda \int_{L_{z}} \exp (-\omega t) u_{, \alpha} v, \alpha \mathrm{d} x_{2}+\left(\rho_{1} k_{1}-\lambda\right) \int_{0}^{t} \int_{L_{z}} \exp (-\omega \eta) u_{, 1 \eta} u_{, 1} \mathrm{~d} x_{2} \mathrm{~d} \eta \\
& -\lambda \int_{0}^{t} \int_{L_{z}} \exp (-\omega \eta) u_{, \eta} u_{11} \mathrm{~d} x_{2} \mathrm{~d} \eta+2 \lambda \int_{0}^{t} \int_{L_{z}} \exp (-\omega \eta) u_{, 11} v_{, \eta} \mathrm{d} x_{2} \mathrm{~d} \eta \\
& +2 \lambda \int_{0}^{t} \int_{L_{z}} \exp (-\omega \eta) u_{, 1} v, 1 \eta \mathrm{d} x_{2} \mathrm{~d} \eta+\lambda \omega \int_{0}^{t} \int_{L_{z}} \exp (-\omega \eta) u_{, 1} v_{1} \mathrm{~d} x_{2} \mathrm{~d} \eta \\
& +\lambda \omega \int_{0}^{t} \int_{L_{z}} \exp (-\omega \eta) u v, 11 \mathrm{~d} x_{2} \mathrm{~d} \eta+\lambda \int_{L_{z}} \exp (-\omega t) u_{, 1} v_{, 1} \mathrm{~d} x_{2} \\
& +\lambda \int_{L_{z}} \exp (-\omega t) u v, 11 \mathrm{~d} x_{2}+(1+\mu \omega) k_{1} \int_{0}^{t} \int_{L_{z}} \exp (-\omega \eta)\left(u_{, \alpha \alpha}\right)^{2} \mathrm{~d} x_{2} \mathrm{~d} \eta \\
& +\mu k_{1} \int_{L_{z}} \exp (-\omega t)\left(u_{, \alpha \alpha}\right)^{2} \mathrm{~d} x_{2}-\rho_{1} k_{1} \int_{0}^{t} \int_{L_{z}} \exp (-\omega \eta) u_{, \beta \eta} u_{, \beta \eta} \mathrm{d} x_{2} \mathrm{~d} \eta \\
& +\frac{\rho_{2} \omega}{2} \int_{0}^{t} \int_{L_{z}} \exp (-\omega \eta) v_{, \eta}^{2} \mathrm{~d} x_{2} \mathrm{~d} \eta+\frac{\rho_{1}}{2} \int_{L_{z}} \exp (-\omega t) v_{, t}^{2} \mathrm{~d} x_{2} \\
& +\frac{m^{2}}{k} \int_{0}^{t} \int_{L_{z}} \exp (-\omega \eta) v_{, \alpha \eta} v_{, \alpha \eta} \mathrm{d} x_{2} \mathrm{~d} \eta+\frac{\omega \gamma}{2} \int_{0}^{t} \int_{L_{z}} \exp (-\omega t) v_{, \alpha \beta} v_{, \alpha \beta} \mathrm{d} x_{2} \mathrm{~d} \eta \\
& +\frac{\gamma}{2} \int_{L_{z}} \exp (-\omega t) v_{, \alpha \beta} v_{, \alpha \beta} \mathrm{d} x_{2}+\frac{2 \gamma k \rho_{2} \omega}{m^{2}} \int_{0}^{t} \int_{L_{z}} \exp (-\omega \eta) v_{, 1 \eta} v_{1} \mathrm{~d} x_{2} \mathrm{~d} \eta \\
& +\left(\frac{2 \gamma k \rho_{2} \omega}{m^{2}}-\rho_{2} \omega k_{2}\right) \int_{0}^{t} \int_{L_{z}} \exp (-\omega \eta) v, \eta v, 11 \mathrm{~d} x_{2} \mathrm{~d} \eta+\frac{2 \gamma k \rho_{2}}{m^{2}} \int_{L_{z}} \exp (-\omega t) v, 1 t v, 1 \mathrm{~d} x_{2} \\
& +\left(\frac{2 \gamma k \rho_{2}}{m^{2}}+\rho_{2} k_{2}\right) \int_{L_{z}} \exp (-\omega t) v, t v, 11 \mathrm{~d} x_{2}-\frac{2 k \lambda \gamma}{m^{2}} \int_{0}^{t} \int_{L_{z}} \exp (-\omega \eta) u_{, 1 \alpha} v, 1 \alpha \mathrm{d} x_{2} \mathrm{~d} \eta \\
& +\left(\lambda k_{2}-\frac{2 k \lambda \gamma}{m^{2}}\right) \int_{0}^{t} \int_{L_{z}} \exp (-\omega \eta) u_{, \alpha} v, 11 \alpha \mathrm{d} x_{2} \mathrm{~d} \eta-\rho_{2} k_{2} \int_{0}^{t} \int_{L_{z}} \exp (-\omega \eta) v_{, 1 \eta}^{2} \mathrm{~d} x_{2} \mathrm{~d} \eta \\
& +\frac{m^{2}}{2 k} k_{2} \int_{L_{z}} \exp (-\omega t) v_{, 1 \alpha} v_{, 1 \alpha} \mathrm{d} x_{2}+\gamma k_{2} \int_{0}^{t} \int_{L_{z}} \exp (-\omega \eta) v_{, 1 \alpha \beta} v_{, 1 \alpha \beta} \mathrm{d} x_{2} \mathrm{~d} \eta \\
& -\lambda k_{2} \int_{0}^{t} \int_{L_{z}} \exp (-\omega \eta) v_{, 111} u_{, 1} \mathrm{~d} x_{2} \mathrm{~d} \eta-\lambda k_{2} \int_{0}^{t} \int_{L_{z}} \exp (-\omega \eta) v, 11 u_{, 11} \mathrm{~d} x_{2} \mathrm{~d} \eta \\
& +k_{2} \int_{0}^{t} \int_{L_{z}} \exp (-\omega \eta) v_{, 112} v, 2 \eta \mathrm{d} x_{2} \mathrm{~d} \eta+k_{2} \int_{0}^{t} \int_{L_{z}} \exp (-\omega \eta) v_{, 12} u_{, 12 \eta} \mathrm{d} x_{2} \mathrm{~d} \eta \text {. }
\end{aligned}
$$

Using the results (3.1)-(3.3) in [19], we have

$$
\begin{gathered}
\int_{0}^{t} \int_{L_{z}} \exp (-\omega \eta) v_{, \alpha} v_{, \alpha} \mathrm{d} x_{2} \mathrm{~d} \eta \leq c_{1} \int_{0}^{t} \int_{L_{z}} \exp (-\omega \eta) v_{, \alpha \beta} v_{, \alpha \beta} \mathrm{d} x_{2} \mathrm{~d} \eta, \\
\int_{L_{z}} \exp (-\omega t) v_{, \alpha} v_{, \alpha} \mathrm{d} x_{2} \leq c_{1} \int_{L_{z}} \exp (-\omega t) v_{, \alpha \beta} v_{, \alpha \beta} \mathrm{d} x_{2}, \\
\int_{0}^{t} \int_{L_{z}} \exp (-\omega \eta) u^{2} \mathrm{~d} x_{2} \mathrm{~d} \eta \leq c_{2} \int_{0}^{t} \int_{L_{z}} \exp (-\omega \eta) u_{, \alpha \alpha} u_{, \alpha \alpha} \mathrm{d} x_{2} \mathrm{~d} \eta, \\
\int_{L_{z}} \exp (-\omega t) u^{2} \mathrm{~d} x_{2} \leq c_{2} \int_{L_{z}} \exp (-\omega t) u_{, \alpha \alpha} u_{, \alpha \alpha} \mathrm{d} x_{2},
\end{gathered}
$$




$$
\int_{L_{z}} \exp (-\omega t) v_{, 1} v, 1 \mathrm{~d} x_{2} \leq c_{3} \int_{L_{z}} \exp (-\omega t) v_{, 1 \alpha} v, 1 \alpha \mathrm{d} x_{2} \mathrm{~d} \eta,
$$

with $c_{1}, c_{2}$, and $c_{3}$ are positive constants .

Using the Schwarz inequality, and combining (37)-(42), we obtain

$$
\begin{aligned}
& \frac{\partial^{2} \varphi(z, t)}{\partial z^{2}} \geq\left(\frac{\omega \rho_{1}}{2}-\frac{\lambda}{2} \varepsilon_{4}\right) \int_{0}^{t} \int_{L_{z}} \exp (-\omega \eta) u_{, \eta}^{2} \mathrm{~d} x_{2} \mathrm{~d} \eta+\frac{\rho_{1}}{2} \int_{L_{z}} \exp (-\omega t) u_{, t}^{2} \mathrm{~d} x_{2} \\
& +\left[\frac{\omega}{2}-\frac{\lambda \omega}{2} \varepsilon_{1}-\frac{\rho_{1} k_{1}-\lambda}{2 \varepsilon_{3}}-\lambda \varepsilon_{6}-\frac{\lambda \omega}{2} \varepsilon_{7}-\frac{k \lambda \gamma}{m^{2}} \varepsilon_{15}-\left(\lambda k_{2}-\frac{2 k \lambda \gamma}{m^{2}}\right) \frac{\varepsilon_{16}}{2}\right. \\
& \left.-\frac{\lambda k_{2}}{2 \varepsilon_{17}}\right] \int_{0}^{t} \int_{L_{z}} \exp (-\omega \eta) u_{, \alpha} u_{, \alpha} \mathrm{d} x_{2} \mathrm{~d} \eta+\left(\frac{1}{2}-\frac{\lambda}{2} \varepsilon_{2}-\frac{\lambda}{2} \varepsilon_{9}\right) \int_{L_{z}} \exp (-\omega t) u_{, \alpha} u, \alpha \mathrm{d} x_{2} \\
& +\left[\mu-\frac{\rho_{1} k_{1}-\lambda}{2} \varepsilon_{3}-\rho_{1} k_{1}\right] \int_{0}^{t} \int_{L_{z}} \exp (-\omega \eta) u_{, \alpha \eta} u_{, \alpha \eta} \mathrm{d} x_{2} \mathrm{~d} \eta \\
& +\left[(1+\mu \omega) k_{1}-\frac{1}{2 \varepsilon_{4}}-\lambda \varepsilon_{5}-\frac{\lambda \omega c_{2}}{2} \varepsilon_{8}-\frac{k \lambda \gamma}{m^{2}} \varepsilon_{14}-\frac{\lambda k_{2}}{2 \varepsilon_{18}}\right] \int_{0}^{t} \int_{L_{z}} \exp (-\omega \eta)\left(u_{, \alpha \eta}\right)^{2} \mathrm{~d} x_{2} \mathrm{~d} \eta \\
& +\left(\mu k_{1}-\frac{\lambda c_{2}}{2} \varepsilon_{10}\right) \int_{L_{z}} \exp (-\omega t)\left(u_{, \alpha \alpha}\right)^{2} \mathrm{~d} x_{2} \\
& +\left[\frac{\rho_{2} \omega}{2}-\frac{\lambda}{\varepsilon_{5}}-\frac{1}{2}\left(\frac{2 \gamma k \rho_{2} \omega}{m^{2}}-\rho_{2} \omega k_{2}\right) \varepsilon_{12}\right] \int_{0}^{t} \int_{L_{z}} \exp (-\omega \eta) v_{, \eta}^{2} \mathrm{~d} x_{2} \mathrm{~d} \eta \\
& +\left[\frac{\rho_{2}}{2}-\frac{1}{2}\left(\frac{2 \gamma k \rho_{2}}{m^{2}}+\rho_{2} k_{2}\right) \varepsilon_{13}\right] \int_{L_{z}} \exp (-\omega t) v_{, t}^{2} \mathrm{~d} x_{2} \\
& +\left[\frac{m^{2}}{k}-\frac{\lambda}{\varepsilon_{6}}-\frac{\gamma k \rho_{2} \omega}{m^{2}} \varepsilon_{11}-\frac{k_{2}}{2 \varepsilon_{19}}-\rho_{2} k_{2}\right] \int_{0}^{t} \int_{L_{z}} \exp (-\omega \eta) v, \alpha \eta v, \alpha \eta \mathrm{d} x_{2} \mathrm{~d} \eta \\
& +\left[\frac{\omega \gamma}{2}-\frac{\lambda \omega c_{1}}{2 \varepsilon_{1}}-\frac{\lambda \omega c_{1}}{2 \varepsilon_{7}}-\frac{\lambda \omega}{2 \varepsilon_{8}}-\frac{\gamma k \rho_{2} \omega c_{1}}{m^{2} \varepsilon_{11}}-\frac{1}{2}\left(\frac{2 \gamma k \rho_{2} \omega}{m^{2}}-\rho_{2} \omega k_{2}\right) \frac{1}{\varepsilon_{12}}-\frac{k \lambda \gamma}{m^{2}} \frac{1}{\varepsilon_{14}}\right. \\
& \left.-\frac{\lambda k_{2}}{2} \varepsilon_{18}\right] \int_{0}^{t} \int_{L_{z}} \exp (-\omega \eta) v_{, \alpha \beta} v_{, \alpha \beta} \mathrm{d} x_{2} \mathrm{~d} \eta+\left[\frac{\gamma}{2}-\frac{\lambda c_{1}}{2 \varepsilon_{2}}\right] \int_{L_{z}} \exp (-\omega t) v_{, \alpha \beta} v_{, \alpha \beta} \mathrm{d} x_{2} \\
& +\left[\frac{m^{2}}{2 k} k_{2}-\frac{\lambda c_{3}}{2 \varepsilon_{9}}-\frac{\lambda}{2 \varepsilon_{10}}-\frac{1}{2 \varepsilon_{13}}\left(\frac{2 \gamma k \rho_{2}}{m^{2}}+\rho_{2} k_{2}\right)\right] \int_{L_{z}} \exp (-\omega t) v_{, 1 \alpha} v_{1 \alpha} \mathrm{d} x_{2} \\
& +\left[\gamma k_{2}-\frac{k \lambda \gamma}{m^{2} \varepsilon_{15}}-\left(\lambda k_{2}-\frac{2 k \lambda \gamma}{m^{2}}\right) \frac{1}{2 \varepsilon_{16}}-\frac{\lambda k_{2}}{2} \varepsilon_{17}-\frac{k_{2}}{2} \varepsilon_{19}\right] \int_{0}^{t} \int_{L_{z}} \exp (-\omega \eta) v_{, 1 \alpha \beta} v_{1 \alpha \beta} \mathrm{d} x_{2} \mathrm{~d} \eta \\
& +\frac{k_{2}}{2} \int_{L_{z}} \exp (-\omega t) v, 12 v_{, 12} \mathrm{~d} x_{2}+\frac{2 \gamma k \rho_{2}}{m^{2}} \int_{L_{z}} \exp (-\omega t) v_{, 1 t} v_{, 1} \mathrm{~d} x_{2}
\end{aligned}
$$

where $\varepsilon_{i},(i=1,2, \cdots, 20)$ are arbitrary positive constants .

In (43), if we choose $\varepsilon_{1}=\varepsilon_{2}=\varepsilon_{7}=\varepsilon_{9}=\frac{1}{4 \lambda}, \varepsilon_{3}=2, \varepsilon_{4}=\frac{\omega \rho_{1}}{2 \lambda}, \varepsilon_{5}=\frac{\omega \rho_{2}}{8 \lambda}, \varepsilon_{6}=\lambda, \varepsilon_{8}=1$, $\varepsilon_{10}=\frac{\mu k_{1}}{\lambda c_{2}}, \varepsilon_{11}=\varepsilon_{12}=\gamma, \varepsilon_{13}=\frac{1}{2}\left(\frac{2 \gamma k \rho_{2}}{m^{2}}+\rho_{2} k_{2}\right)^{-1} \rho_{1}, \varepsilon_{14}=\frac{\gamma}{\omega}, \varepsilon_{15}=\frac{k \lambda \gamma}{m^{2}}, \varepsilon_{16}=\frac{2 \lambda}{\gamma}, \varepsilon_{17}=\frac{\gamma}{2 \lambda}$, $\varepsilon_{18}=\omega, \varepsilon_{19}=\frac{\gamma}{2}, k_{1}=\frac{1}{2 \rho_{1}}\left(\frac{\mu}{2}+\lambda\right), k_{2}=\frac{4}{\gamma} ; m \geq \max \left\{\sqrt{\frac{4 k}{k_{2}}\left[2 \lambda^{2} c_{3}+\frac{\lambda^{2} c_{2}}{2 \mu k_{1}}+\frac{\left(2 \gamma k \rho_{2}+\rho_{2} k_{2}\right)^{2}}{\rho_{1}}\right]}\right.$, $\left.1,8 \gamma^{2} k, \sqrt{2 k\left(1+\gamma^{2} k \rho_{2} \omega+\frac{4}{\gamma^{2}}+\frac{4 \rho_{2}}{\gamma}\right)}\right\}, \omega \geq \max \left\{2 k_{1} \rho_{1}+16 \lambda^{2}+8 k^{2} \lambda^{2} \gamma^{2}, 1\right\}, \mu \geq$ $\sqrt{\frac{8 \lambda}{\omega^{2}}+\rho_{1} \rho_{2}+4 \rho_{1} \lambda c_{1}+8 \rho_{1} k \lambda \gamma^{2}}, \gamma \geq \max \left\{16 \lambda^{2} c_{1}+10 \lambda+4 k \rho_{2} c_{1}+4 k \rho_{2}+4 k \lambda, 1\right\}$, we can get 


$$
\begin{aligned}
\frac{\partial^{2} \varphi(z, t)}{\partial z^{2}} & \geq \frac{\omega \rho_{1}}{4} \int_{0}^{t} \int_{L_{z}} \exp (-\omega \eta) u_{, \eta}^{2} \mathrm{~d} x_{2} \mathrm{~d} \eta+\frac{\rho_{1}}{2} \int_{L_{z}} \exp (-\omega t) u_{, t}^{2} \mathrm{~d} x_{2} \\
& +\frac{\omega}{8} \int_{0}^{t} \int_{L_{z}} \exp (-\omega \eta) u_{, \alpha} u_{, \alpha} \mathrm{d} x_{2} \mathrm{~d} \eta+\frac{1}{4} \int_{L_{z}} \exp (-\omega t) u_{, \alpha} u_{, \alpha} \mathrm{d} x_{2} \\
& +\frac{\mu}{2} \int_{0}^{t} \int_{L_{z}} \exp (-\omega \eta) u_{, \alpha \eta} u_{, \alpha \eta} \mathrm{d} x_{2} \mathrm{~d} \eta+\frac{\mu \omega k_{1}}{2} \int_{0}^{t} \int_{L_{z}} \exp (-\omega \eta)\left(u_{, \alpha \alpha}\right)^{2} \mathrm{~d} x_{2} \mathrm{~d} \eta \\
& +\frac{\mu k_{1}}{2} \int_{L_{z}} \exp (-\omega t)\left(u_{, \alpha \alpha}\right)^{2} \mathrm{~d} x_{2}+\frac{\rho_{2} \omega}{4} \int_{0}^{t} \int_{L_{z}} \exp (-\omega \eta) v_{, \eta}^{2} \mathrm{~d} x_{2} \mathrm{~d} \eta \\
& +\frac{\rho_{2}}{4} \int_{L_{z}} \exp (-\omega t) v_{, t}^{2} \mathrm{~d} x_{2}+\frac{m^{2}}{2 k} \int_{0}^{t} \int_{L_{z}} \exp (-\omega \eta) v_{, \alpha \eta} v_{, \alpha \eta} \mathrm{d} x_{2} \mathrm{~d} \eta \\
& +\frac{\omega \gamma}{4} \int_{0}^{t} \int_{L_{z}} \exp (-\omega \eta) v_{, \alpha \beta} v_{, \alpha \beta} \mathrm{d} x_{2} \mathrm{~d} \eta+\frac{\gamma}{4} \int_{L_{z}} \exp (-\omega t) v_{, \alpha \beta} v_{, \alpha \beta} \mathrm{d} x_{2} \\
& +\frac{m^{2}}{4 k} k_{2} \int_{L_{z}} \exp (-\omega t) v_{, 1 \alpha} v_{, 1 \alpha} \mathrm{d} x_{2}+\int_{0}^{t} \int_{L_{z}} \exp (-\omega \eta) v_{, 1 \alpha \beta} v_{, 1 \alpha \beta} \mathrm{d} x_{2} \mathrm{~d} \eta \\
& +\frac{k_{2}}{2} \int_{L_{z}} \exp (-\omega t) v_{12} v_{, 12} \mathrm{~d} x_{2}+\frac{2 \gamma k \rho_{2}}{m^{2}} \int_{L_{z}} \exp (-\omega t) v_{, 1 t} v_{, 1} \mathrm{~d} x_{2} \\
& =E(z, t) .
\end{aligned}
$$

We now define a new function $F(z, t)$ as

$$
F(z, t)=\int_{0}^{t} E(z, s) d s .
$$

We get

$$
\begin{aligned}
F(z, t) & =\frac{\omega \rho_{1}}{4} \int_{0}^{t} \int_{0}^{s} \int_{L_{z}} \exp (-\omega \eta) u_{, \eta}^{2} \mathrm{~d} x_{2} \mathrm{~d} \eta d s+\frac{\rho_{1}}{2} \int_{0}^{t} \int_{L_{z}} \exp (-\omega s) u_{, s}^{2} \mathrm{~d} x_{2} d s \\
& +\frac{\omega}{8} \int_{0}^{t} \int_{0}^{s} \int_{L_{z}} \exp (-\omega \eta) u_{, \alpha} u_{, \alpha} \mathrm{d} x_{2} \mathrm{~d} \eta d s+\frac{1}{4} \int_{0}^{t} \int_{L_{z}} \exp (-\omega s) u_{, \alpha} u_{, \alpha} \mathrm{d} x_{2} d s \\
& +\frac{\mu}{2} \int_{0}^{t} \int_{0}^{s} \int_{L_{z}} \exp (-\omega \eta) u_{, \alpha \eta} u_{, \alpha \eta} \mathrm{d} x_{2} \mathrm{~d} \eta d s+\frac{\mu \omega k_{1}}{2} \int_{0}^{t} \int_{0}^{s} \int_{L_{z}} \exp (-\omega \eta)\left(u_{, \alpha \alpha}\right)^{2} \mathrm{~d} x_{2} \mathrm{~d} \eta d s \\
& +\frac{\mu k_{1}}{2} \int_{0}^{t} \int_{L_{z}} \exp (-\omega s)\left(u_{, \alpha \alpha}\right)^{2} \mathrm{~d} x_{2} d s+\frac{\rho_{2} \omega}{4} \int_{0}^{t} \int_{0}^{s} \int_{L_{z}} \exp (-\omega \eta) v_{, \eta}^{2} \mathrm{~d} x_{2} \mathrm{~d} \eta d s \\
& +\frac{\rho_{2}}{4} \int_{0}^{t} \int_{L_{z}} \exp (-\omega s) v_{, t}^{2} \mathrm{~d} x_{2} d s+\frac{m^{2}}{2 k} \int_{0}^{t} \int_{0}^{s} \int_{L_{z}} \exp (-\omega \eta) v, \alpha \eta v_{, \alpha \eta} \mathrm{d} x_{2} \mathrm{~d} \eta d s \\
& +\frac{\omega \gamma}{4} \int_{0}^{t} \int_{0}^{s} \int_{L_{z}} \exp (-\omega \eta) v_{, \alpha \beta} v_{, \alpha \beta} \mathrm{d} x_{2} \mathrm{~d} \eta d s+\frac{\gamma}{4} \int_{0}^{t} \int_{L_{z}} \exp (-\omega s) v_{, \alpha \beta} v_{, \alpha \beta} \mathrm{d} x_{2} d s \\
& +\frac{m^{2}}{4 k} k_{2} \int_{0}^{t} \int_{L_{z}} \exp (-\omega s) v_{, 1 \alpha} v, 1 \alpha \mathrm{d} x_{2} d s+\int_{0}^{t} \int_{0}^{s} \int_{L_{z}} \exp (-\omega \eta) v_{, 1 \alpha \beta} v_{, 1 \alpha \beta} \mathrm{d} x_{2} \mathrm{~d} \eta d s \\
& +\frac{k_{2}}{2} \int_{0}^{t} \int_{L_{z}} \exp (-\omega s) v_{, 12}^{2} \mathrm{~d} x_{2} d s+\frac{\omega \gamma k \rho_{2}}{m^{2}} \int_{0}^{t} \int_{L_{z}} \exp (-\omega s) v_{, 1}^{2} \mathrm{~d} x_{2} d s \\
& +\frac{\gamma k \rho_{2}}{m^{2}} \int_{L_{z}} \exp (-\omega t) v_{, 1}^{2} \mathrm{~d} x_{2} .
\end{aligned}
$$


We can easily get

$$
F(z, t) \geq 0 .
$$

Following the same procedures as (37)-(44), we obtain

$$
\frac{\partial^{2} \varphi(z, t)}{\partial z^{2}} \leq \frac{3}{2} E(z, t) .
$$

Inserting (30) into (48), we have

$$
\frac{\partial^{2} \Phi(z, t)}{\partial z^{2}} \leq \frac{3}{2} F(z, t) .
$$

We can also get

$$
\frac{\partial^{2} \Phi(z, t)}{\partial z^{2}} \geq F(z, t) .
$$

Combining (47) and (50), we obtain

$$
\frac{\partial^{2} \Phi(z, t)}{\partial z^{2}}=\frac{\partial^{2} \int_{0}^{t} \varphi(z, s) d s}{\partial z^{2}} \geq 0 .
$$

Combining (36), (44) and (51), using the Schwarz's inequality, we can obtain

$$
|\Phi(z, t)| \leq k_{3} \frac{\partial^{2} \Phi(z, t)}{\partial z^{2}},
$$

where $k_{3}$ is a computable positive constant. The proof of Lemma 5 is finished.

\section{Phragmén-Lindelöf Alternative Results}

Based on Lemmas 1-5, we can get the following Lemmas:

Lemma 6. We suggest $u$ and $v$ are classical solutions of problems (4)-(7) in the semi-infinite strip $\Omega_{0}$ defined by (1), if there exists $a z_{0} \geq 0$ such that $\frac{\partial \Phi\left(z_{0}, t\right)}{\partial z}>0$, then the following inequality holds:

$$
\lim _{z \rightarrow \infty} e^{-k_{4} z} G(z, t) \geq c_{1}(t)
$$

where $c_{1}(t)=\frac{2}{3}\left[\frac{\partial}{\partial z} \Phi\left(z_{1}, t\right)+k_{4} \Phi\left(z_{1}, t\right)\right] e^{-k_{4} z_{1}}, G(z, t)$ will be defined in (61).

Proof. Since $\frac{\partial^{2} \Phi\left(z_{0}, t\right)}{\partial z^{2}} \geq 0$ for all $z \geq 0$, we can get $\frac{\partial \Phi(z, t)}{\partial z}>0$ for all $z \geq z_{0}$.

We know the fact $\Phi(z, t) \geq \Phi\left(z_{0}, t\right)+\frac{\partial \Phi\left(z_{0}, t\right)}{\partial z}\left(z-z_{0}\right)$ for all $z \geq z_{0}$.

If we let $z \rightarrow+\infty$, we can obtain $\Phi(z, t)>0$.

So, wo have the following results:

There exists a $z_{1}>z_{0}$ such that $\frac{\partial \Phi\left(z_{1}, t\right)}{\partial z}>0$ and $\Phi\left(z_{1}, t\right)>0$.

From (31), we can get

$$
\frac{\partial^{2} \Phi(z, t)}{\partial z^{2}}-k_{4}^{2} \Phi(z, t) \geq 0
$$

with $k_{4}=\sqrt{\frac{1}{k_{3}}}$. 
Equation (53) can be rewritten as

$$
\frac{\partial}{\partial z}\left(e^{-k_{4} z}\left[\frac{\partial}{\partial z} \Phi(z, t)+k_{4} \Phi(z, t)\right]\right) \geq 0,
$$

or

$$
\frac{\partial}{\partial z}\left(e^{k_{4} z}\left[\frac{\partial}{\partial z} \Phi(z, t)-k_{4} \Phi(z, t)\right]\right) \geq 0 .
$$

Integrating (54) and (55), we obtain

$$
\frac{\partial}{\partial z} \Phi(z, t)+k_{4} \Phi(z, t) \geq\left[\frac{\partial}{\partial z} \Phi\left(z_{1}, t\right)+k_{4} \Phi\left(z_{1}, t\right)\right] e^{k_{4}\left(z-z_{1}\right)},
$$

or

$$
\frac{\partial}{\partial z} \Phi(z, t)-k_{4} \Phi(z, t) \geq\left[\frac{\partial}{\partial z} \Phi\left(z_{1}, t\right)-k_{4} \Phi\left(z_{1}, t\right)\right] e^{-k_{4}\left(z-z_{1}\right)},
$$

for all $z \geq z_{1}$.

Combining (56) and (57), we have

$$
\begin{aligned}
\frac{\partial}{\partial z} \Phi(z, t) & \geq \frac{\partial}{\partial z} \Phi\left(z_{1}, t\right) \frac{e^{k_{4}\left(z-z_{1}\right)}+e^{-k_{4}\left(z-z_{1}\right)}}{2} \\
& +k_{4} \Phi\left(z_{1}, t\right) \frac{e^{k_{4}\left(z-z_{1}\right)}-e^{-k_{4}\left(z-z_{1}\right)}}{2} .
\end{aligned}
$$

Integrating (49) from $z_{1}$ to $z$, we obtain

$$
\int_{z_{1}}^{z} \frac{\partial^{2} \Phi(z, t)}{\partial z^{2}} d \xi \leq \frac{3}{2} \int_{z_{1}}^{z} F(\xi, t) d \xi .
$$

Inserting (59) into (58), we have

$$
\begin{aligned}
\frac{3}{2} \int_{z_{1}}^{z} F(\xi, t) d \xi & \geq \frac{\partial}{\partial z} \Phi\left(z_{1}, t\right)\left[\frac{e^{k_{4}\left(z-z_{1}\right)}+e^{-k_{4}\left(z-z_{1}\right)}}{2}-1\right] \\
& +k_{4} \Phi\left(z_{1}, t\right) \frac{e^{k_{4}\left(z-z_{1}\right)}-e^{-k_{4}\left(z-z_{1}\right)}}{2} .
\end{aligned}
$$

If we define

$$
G(z, t)=\int_{z_{1}}^{z} F(\xi, t) d \xi
$$


Combining (46) and (61), we have

$$
\begin{aligned}
& G(z, t)=\frac{\omega \rho_{1}}{4} \int_{0}^{t} \int_{0}^{s} \int_{z_{1}}^{z} \int_{L_{\tilde{\zeta}}} \exp (-\omega \eta) u_{, \eta}^{2} \mathrm{~d} A \mathrm{~d} \eta d s \\
& +\frac{\rho_{1}}{2} \int_{0}^{t} \int_{z_{1}}^{z} \int_{L_{\tilde{\xi}}} \exp (-\omega s) u_{, s}^{2} \mathrm{~d} A d s \\
& +\frac{\omega}{8} \int_{0}^{t} \int_{0}^{s} \int_{z_{1}}^{z} \int_{L_{\tilde{\zeta}}} \exp (-\omega \eta) u, \alpha u, \alpha \mathrm{d} A \mathrm{~d} \eta d s \\
& +\frac{1}{4} \int_{0}^{t} \int_{z_{1}}^{z} \int_{L_{\tilde{\zeta}}} \exp (-\omega s) u_{, \alpha} u_{, \alpha} \mathrm{d} A d s \\
& +\frac{\mu}{2} \int_{0}^{t} \int_{0}^{s} \int_{z_{1}}^{z} \int_{L_{\tilde{\xi}}} \exp (-\omega \eta) u_{, \alpha \eta} u_{, \alpha \eta} \mathrm{d} A \mathrm{~d} \eta d s \\
& +\frac{\mu \omega k_{1}}{2} \int_{0}^{t} \int_{0}^{s} \int_{z_{1}}^{z} \int_{L_{\tilde{\xi}}} \exp (-\omega \eta)(u, \alpha \alpha)^{2} \mathrm{~d} A \mathrm{~d} \eta d s \\
& +\frac{\mu k_{1}}{2} \int_{0}^{t} \int_{z_{1}}^{z} \int_{L_{\xi}} \exp (-\omega s)\left(u_{, \alpha \alpha}\right)^{2} \mathrm{~d} A d s \\
& +\frac{\rho_{2} \omega}{4} \int_{0}^{t} \int_{0}^{s} \int_{z_{1}}^{z} \int_{L_{\tilde{\xi}}} \exp (-\omega \eta) v_{, \eta}^{2} \mathrm{~d} A \mathrm{~d} \eta d s \\
& +\frac{\rho_{2}}{4} \int_{0}^{t} \int_{z_{1}}^{z} \int_{L_{\xi}} \exp (-\omega s) v_{, t}^{2} \mathrm{~d} A d s \\
& +\frac{m^{2}}{2 k} \int_{0}^{t} \int_{0}^{s} \int_{z_{1}}^{z} \int_{L_{\tilde{\zeta}}} \exp (-\omega \eta) v_{, \alpha \eta} v_{, \alpha \eta} \mathrm{d} A \mathrm{~d} \eta d s \\
& +\frac{\omega \gamma}{4} \int_{0}^{t} \int_{0}^{s} \int_{z_{1}}^{z} \int_{L_{\tilde{\zeta}}} \exp (-\omega \eta) v_{, \alpha \beta} v_{, \alpha \beta} \mathrm{d} A \mathrm{~d} \eta d s
\end{aligned}
$$

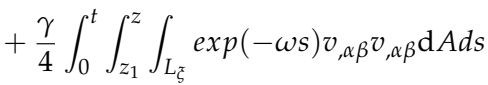

$$
\begin{aligned}
& +\frac{m^{2}}{4 k} k_{2} \int_{0}^{t} \int_{z_{1}}^{z} \int_{L_{\tilde{\xi}}} \exp (-\omega s) v_{, 1 \alpha} v, 1 \alpha \mathrm{d} A d s
\end{aligned}
$$

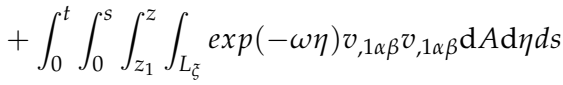

$$
\begin{aligned}
& +\frac{k_{2}}{2} \int_{0}^{t} \int_{z_{1}}^{z} \int_{L_{\tilde{\xi}}} \exp (-\omega s) v_{, 12}^{2} \mathrm{~d} A d s \\
& +\frac{\omega \gamma k \rho_{2}}{m^{2}} \int_{0}^{t} \int_{z_{1}}^{z} \int_{L_{\tilde{\xi}}} \exp (-\omega s) v_{, 1}^{2} \mathrm{~d} A d s \\
& +\frac{\gamma k \rho_{2}}{m^{2}} \int_{z_{1}}^{z} \int_{L_{\tilde{\zeta}}} \exp (-\omega t) v_{, 1}^{2} \mathrm{~d} A \text {. }
\end{aligned}
$$

we obtain

$$
\lim _{z \rightarrow \infty} e^{-k_{4} z} G(z, t) \geq c_{1}(t)
$$

with $c_{1}(t)=\frac{2}{3}\left[\frac{\partial}{\partial z} \Phi\left(z_{1}, t\right)+k_{4} \Phi\left(z_{1}, t\right)\right] e^{-k_{4} z_{1}}$.

Lemma 7. We suggest $u$ and $v$ are classical solutions of problems (4)-(7) in the semi-infinite strip $\Omega_{0}$ defined by (1). If $\frac{\partial \Phi(z, t)}{\partial z} \leq 0$ for all $z \geq 0$, then the following inequality holds:

$$
H(z, t) \leq c_{2}(t) e^{-k_{4} z} .
$$

where $\left.c_{2}(t)=-\frac{\partial}{\partial z} \Phi(0, t)+k_{4} \Phi(0, t)\right], H(z, t)$ will be defined in (66).

Proof. If we suggest there exists a $z_{0}>0$, such that $\Phi\left(z_{0}, t\right)<0$. Since $\frac{\partial \Phi(z, t)}{\partial z} \leq 0$ for all $z \geq 0$, we can get $\Phi(z, t) \leq \Phi\left(z_{0}, t\right)<0$ for all $z \geq z_{0}$. From (31), we have $\frac{\partial \Phi(z, t)}{\partial z}-\frac{\partial \Phi\left(z_{0}, t\right)}{\partial z}=\frac{\partial^{2} \Phi(\xi, t)}{\partial z}\left(z-z_{0}\right) \geq-\frac{1}{k_{3}} \Phi(\xi, t)\left(z-z_{0}\right)$, with $z_{0}<\xi<z$. let $z \rightarrow \infty$, we have $\frac{\partial \Phi(z, t)}{\partial z}>0$. Which gives a contradiction to $\frac{\partial \Phi(z, t)}{\partial z} \leq 0$ for all $z \geq 0$. So we can conclude $\Phi(z, t) \geq 0$ for all $z \geq 0$. 
Integrating (53) from 0 to $z$, we obtain

$$
-\frac{\partial \Phi(z, t)}{\partial z}+k_{4} \Phi(z, t) \leq c_{2}(t) e^{-k_{4} z}
$$

with $\left.c_{2}(t)=-\frac{\partial}{\partial z} \Phi(0, t)+k_{4} \Phi(0, t)\right]$.

Since $\Phi(z, t) \geq 0$ for for all $z \geq 0$, we have

$$
-\frac{\partial \Phi(z, t)}{\partial z} \leq c_{2}(t) e^{-k_{4} z}
$$

From (64), we can get the results $\Phi(z, t)$ and $-\frac{\partial \Phi(z, t)}{\partial z}$ tend to 0 as $z \rightarrow \infty$. We thus have

$$
\begin{aligned}
-\frac{\partial \Phi(z, t)}{\partial z} & =\int_{z}^{\infty} \frac{\partial^{2} \Phi(\xi, t)}{\partial z^{2}} d \xi \\
& \geq \int_{z}^{\infty} F(\xi, t) d \xi \\
& =H(z, t) .
\end{aligned}
$$

Combining (46) and (66), we have

$$
\begin{aligned}
& H(z, t)=\frac{\omega \rho_{1}}{4} \int_{0}^{t} \int_{0}^{s} \int_{z}^{\infty} \int_{L_{\tilde{\xi}}} \exp (-\omega \eta) u_{, \eta}^{2} \mathrm{~d} A \mathrm{~d} \eta d s \\
& +\frac{\rho_{1}}{2} \int_{0}^{t} \int_{z}^{\infty} \int_{L_{\xi}} \exp (-\omega s) u_{, s}^{2} \mathrm{~d} A d s \\
& +\frac{\omega}{8} \int_{0}^{t} \int_{0}^{s} \int_{z}^{\infty} \int_{L_{\xi}} \exp (-\omega \eta) u_{, \alpha} u_{, \alpha} \mathrm{d} A \mathrm{~d} \eta d s \\
& +\frac{1}{4} \int_{0}^{t} \int_{z}^{\infty} \int_{L_{\tilde{\zeta}}} \exp (-\omega s) u_{, \alpha} u_{, \alpha} \mathrm{d} x_{2} d s \\
& +\frac{\mu}{2} \int_{0}^{t} \int_{0}^{s} \int_{z}^{\infty} \int_{L_{\tilde{\xi}}} \exp (-\omega \eta) u_{, \alpha \eta} u, \alpha \eta \mathrm{d} A \mathrm{~d} \eta d s \\
& +\frac{\mu \omega k_{1}}{2} \int_{0}^{t} \int_{0}^{s} \int_{z}^{\infty} \int_{L_{\xi}} \exp (-\omega \eta)(u, \alpha \alpha)^{2} \mathrm{~d} A \mathrm{~d} \eta d s \\
& +\frac{\mu k_{1}}{2} \int_{0}^{t} \int_{z}^{\infty} \int_{L_{\xi}} \exp (-\omega s)\left(u_{, \alpha \alpha}\right)^{2} \mathrm{~d} A d s \\
& +\frac{\rho_{2} \omega}{4} \int_{0}^{t} \int_{0}^{s} \int_{z}^{\infty} \int_{L_{\tilde{\xi}}} \exp (-\omega \eta) v_{, \eta}^{2} \mathrm{~d} A \mathrm{~d} \eta d s \\
& +\frac{\rho_{2}}{4} \int_{0}^{t} \int_{z}^{\infty} \int_{L_{\xi}} \exp (-\omega s) v_{, t}^{2} \mathrm{~d} A d s \\
& +\frac{m^{2}}{2 k} \int_{0}^{t} \int_{0}^{s} \int_{z}^{\infty} \int_{L_{\tilde{\xi}}} \exp (-\omega \eta) v, \alpha \eta v, \alpha \eta \mathrm{d} A \mathrm{~d} \eta d s \\
& +\frac{\omega \gamma}{4} \int_{0}^{t} \int_{0}^{s} \int_{z}^{\infty} \int_{L_{\tilde{\xi}}} \exp (-\omega \eta) v_{, \alpha \beta} v_{, \alpha \beta} \mathrm{d} A \mathrm{~d} \eta d s \\
& +\frac{\gamma}{4} \int_{0}^{t} \int_{z}^{\infty} \int_{L_{\xi}} \exp (-\omega s) v, \alpha \beta v, \alpha \beta \mathrm{d} A d s \\
& +\frac{m^{2}}{4 k} k_{2} \int_{0}^{t} \int_{z}^{\infty} \int_{L_{\tilde{\xi}}} \exp (-\omega s) v_{, 1 \alpha} v, 1 \alpha \mathrm{d} A d s
\end{aligned}
$$

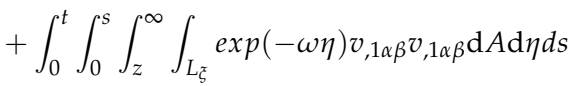

$$
\begin{aligned}
& +\frac{k_{2}}{2} \int_{0}^{t} \int_{z}^{\infty} \int_{L_{\tilde{\xi}}} \exp (-\omega s) v_{, 12}^{2} \mathrm{~d} A d s \\
& +\frac{\omega \gamma k \rho_{2}}{m^{2}} \int_{0}^{t} \int_{z}^{\infty} \int_{L_{\tilde{\xi}}} \exp (-\omega s) v_{, 1}^{2} \mathrm{~d} A d s \\
& +\frac{\gamma k \rho_{2}}{m^{2}} \int_{z}^{\infty} \int_{L_{\tilde{\xi}}} \exp (-\omega t) v_{, 1}^{2} \mathrm{~d} A .
\end{aligned}
$$


Inserting (66) into (65), we obtain

$$
H(z, t) \leq c_{2}(t) e^{-k_{4} z}
$$

Based on Lemmas 6 and 7, we can get the following theorem.

Theorem 1. We suggest $u$ and $v$ are classical solutions of problems (4)-(7) in the semi-infinite strip $\Omega_{0}$ defined by (1), then either inequality

$$
\lim _{z \rightarrow \infty} e^{-k_{4} z} G(z, t) \geq c_{1}(t)
$$

holds or

$$
H(z, t) \leq c_{2}(t) e^{-k_{4} z}
$$

holds.

Theorem 1 shows that either the energy expression $G(z, t)$ grows exponentially or the energy expression $H(z, t)$ decays exponentially.

\section{Conclusions}

In this paper, we studied the spatial properties of solutions for a class of thermoelastic plate with biharmonic operator in a semi-infinite cylinder in $R^{2}$. The Phragmén-Lindelöf alternative results were obtained based on a second-order inequality. Our method is also valid for the hyperbolic-parabolic coupling equations. We can only deal with the linear equations. For the case of nonlinear equations, it is difficult to study the spatial properties. The results of these future studies will be of great interest to researchers in our field.

Author Contributions: Writing-original draft, S.L., original draft preparation J.S. and review and editing, B.O. All authors have read and agreed to the published version of the manuscript.

Funding: The work was supported national natural Science Foundation of China(Grant \# 61907010), natural Science in Higher Education of Guangdong, China (Grant \# 2018KZDXM048), the General Project of Science Research of Guangzhou (Grant \# 201707010126). Guangdong Province Educational Science "Thirteenth Five-Year Plan" 2020 research project approval (NO. 2020JKDY040).

Data Availability Statement: This paper focuses on theoretical analysis, not involving experiments and data.

Acknowledgments: The authors express their heartfelt thanks to the editors and referees who have provided some important suggestions.

Conflicts of Interest: The authors declare no conflict of interest.

\section{References}

1. Horgan, C.O.; Knowles, J.K. Recent development concerning Saint-Venant's principle. Adv. Appl. Mech. 1983, $23,179-269$.

2. Horgan, C.O. Recent development concerning Saint-Venant's principle: An update. Appl. Mech. Rev. 1989, 42, 295-303. [CrossRef]

3. Horgan, C.O. Recent development concerning Saint-Venant's principle: An second update. Appl. Mech. Rev. 1996, 49, 101-111. [CrossRef]

4. D'Apice, C. Convexity considerations and spatial behavior for the harmonic vibrations in thermoelastic plates. J. Math. Anal. Appl. 2005, 312, 44-60. [CrossRef]

5. D'Apice, C. On a generalized biharmonic equation in plane polars with applications to functionally graded material. Aust. J. Math. Anal. Appl. 2006, 3, 1-15.

6. Chirita, S.; D'Apice, C. On spatial growth or decay of solutions to a non simple heat conduction problem in a semi-infinite strip. An. Stiintifice Univ. Alexandru Ioan Cuza Iasi Mat. 2002, 48, 75-100. 
7. Chirita, S.; Ciarletta, M.; Fabrizio, M. Some spatial decay estimates in time-dependent Stokes slow flows. Appl. Anal. 2001, 77, 211-231. [CrossRef]

8. Fabrizio, M.; Chirita, S. Some qualitative results on the dynamic viscoelasticity of the Reissner-Mindlin plate model. Q. J. Mech. Appl. Math. 2004, 57, 59-78. [CrossRef]

9. Li, Y.F.; Lin, C.H. Spatial Decay for Solutions to 2-D Boussinesq System with Variable Thermal Diffusivity. Acta Appl. Math. 2018, 154, 111-130. [CrossRef]

10. Borrelli, A.; Patria, M.C. Energy bounds in dynamical problems for a semi-infinite magnetoelastic beam. J. Appl. Math. Phys. ZAMP 1996, 47, 880-893. [CrossRef]

11. Diaz, J.I.; Quintallina, R. Spatial and continuous dependence estimates in linear viscoelasticity. J. Math. Anal. Appl. 2002, 273, 1-16. [CrossRef]

12. Quintanilla, R. A spatial decay estimate for the hyperbolic heat equation. SIAM J. Math. Anal. 1998, 27, 78-91. [CrossRef]

13. Quintanilla, R. Phragmen-Lindelof alternative in nonlinear viscoelasticity. Nonlinear Anal. 1998, 14, 7-16. [CrossRef]

14. Payne, L.E.; Schaefer, P.W. Some Phragmén-Lindelöf Type Results for the Biharmonic Equation. J. Appl. Math. Phys. ZAMP 1994, $45,414-432$.

15. Lin, C.H. Spatial Decay Estimates and Energy Bounds forth Stokes Flow Equation. Stab. Appl. Anal. Contin. Media 1992, 2, 249-264.

16. Knowles, J.K. An Energy Estimate for the Biharmonic Equationand its Application to Saint-Venant's Principle in Plane elasto statics. Indian J. Pure Appl. Math. 1983, 14, 791-805.

17. Flavin, J.N. On Knowles' version of Saint-Venant's Principle in Two-dimensional Elastostatics. Arch. Ration. Mech. Anal. 1973, 53, 366-375. [CrossRef]

18. Horgan, C.O. Decay Estimates for the Biharmonic Equation with Applications to Saint-Venant's Principles in Plane Elasticity and Stokes flows. Q. Appl. Math. 1989, 47, 147-157. [CrossRef]

19. Liu, Y.; Lin, C.H. Phragmén-Lindelöftype alternativeresults for the stokes flow equation. Math. Inequalities Appl. 2006, 9, 671-694. [CrossRef]

20. Chen, W.; Palmieri, A. Nonexistence of global solutions for the semilinear Moore-Gibson-Thompson equation in the conservative case. Discret. Contin. Dyn. Syst. 2020, 40, 5513-5540. [CrossRef]

21. Chen, W.; Ikehata, R. The Cauchy problem for the Moore-Gibson-Thompson equation in the dissipative case. J. Differ. Equ. 2021, 292, 176-219. [CrossRef]

22. Palmieri, A.; Takamura, H. Blow-up for a weakly coupled system of semilinear damped wave equations in the scattering case with power nonlinearities. Nonlinear Anal. 2019, 187, 467-492. [CrossRef]

23. Palmieri, A.; Reissig, M. Semi-linear wave models with power non-linearity and scale-invariant time-dependent mass and dissipation, II. Math. Nachr. 2018, 291, 1859-1892. [CrossRef]

24. Liu, Y.; Chen, W. Asymptotic profiles of solutions for regularity-loss-type generalized thermoelastic plate equations and their applications. Z. Angew. Math. Phys. 2020, 71, 1-14. [CrossRef]

25. Liu, Y.; Li, Y.; Shi, J. Estimates for the linear viscoelastic damped wave equation on the Heisenberg group. J. Differ. Equ. 2021, 285, 663-685. [CrossRef]

26. Liu, Y.; Chen, Y.; Luo, C.; Lin, C. Phragmén-Lindelöf alternative results for the shallow water equations for transient compressible viscous flow. J. Math. Anal. Appl. 2013, 398, 409-420. [CrossRef]

27. Liu, Y. Continuous dependence for a thermal convection model with temperature-dependent solubility. Appl. Math. Comput. 2017, 308, 18-30. [CrossRef]

28. Liu, Y.; Xiao, S. Structural stability for the Brinkman fluid interfacing with a Darcy fluid in an unbounded domain. Nonlinear Anal. Real World Appl. 2018, 42, 308-333. [CrossRef]

29. Liu, Y.; Xiao, S.; Lin, Y. Continuous dependence for the Brinkman-Forchheimer fluid interfacing with a Darcy fluid in a bounded domain. Math. Comput. Simul. 2018, 150, 66-82. [CrossRef]

30. Santos, M.L.; Munoz Rivera, J.E. Analytic property of a coupled system of wave-plate type with thermal effect. Differ. Integral Equ. 2011, 24, 965-972.

31. Love, A.E.H. Mathematical Theory of Elasticity, 4th ed.; Dover Publications: New York, NY, USA, 1942. 